TRANSACTIONS OF THE

AMERICAN MATHEMATICAL SOCIETY

Volume 352, Number 10, Pages 4641-4676

S 0002-9947(00)02503-4

Article electronically published on June 14, 2000

\title{
INVARIANT FOLIATIONS NEAR NORMALLY HYPERBOLIC INVARIANT MANIFOLDS FOR SEMIFLOWS
}

\author{
PETER W. BATES, KENING LU, AND CHONGCHUN ZENG
}

\begin{abstract}
Let $M$ be a compact $C^{1}$ manifold which is invariant and normally hyperbolic with respect to a $C^{1}$ semiflow in a Banach space. Then in an $\epsilon$-neighborhood of $M$ there exist local $C^{1}$ center-stable and center-unstable manifolds $W^{c s}(\epsilon)$ and $W^{c u}(\epsilon)$, respectively. Here we show that $W^{c s}(\epsilon)$ and $W^{c u}(\epsilon)$ may each be decomposed into the disjoint union of $C^{1}$ submanifolds (leaves) in such a way that the semiflow takes leaves into leaves of the same collection. Furthermore, each leaf intersects $M$ in a single point which determines the asymptotic behavior of all points of that leaf in either forward or backward time.
\end{abstract}

\section{INTRODUCTION}

This paper, which is a sequel to [BLZ1, is devoted to the existence of stable and unstable invariant foliations for a semiflow in a Banach space. We consider a $C^{1}$ semiflow defined on a Banach space $X$; that is, it is continuous on $[0, \infty) \times X$, and for each $t \geq 0, T^{t}: X \rightarrow X$ is $C^{1}$, and

$$
T^{t} \circ T^{s}(x)=T^{t+s}(x)
$$

for all $t, s \geq 0$ and $x \in X$. A typical example is the solution operator for a differential equation. In [BLZ1] we proved that a compact normally hyperbolic invariant manifold $M$ persists under small $C^{1}$ perturbations in the semiflow. We also showed that in an $\epsilon$ neighborhood of $M$, there exist a center-stable manifold $W^{c s}(\epsilon)$ and a center-unstable manifold $W^{c u}(\epsilon)$ which intersect in the manifold $M$. The purpose of this paper is to show that the center-stable manifold $W^{c s}(\epsilon)$ may be decomposed into an invariant foliation with stable leaves, while the center-unstable manifold $W^{c u}(\epsilon)$ may be decomposed into an invariant foliation with unstable leaves. These fibers or leaves give qualitative properties of the semiflow near $M$.

As an example, let us consider a linear system in $\mathbb{R}^{m+n}$

$$
\begin{array}{ll}
\dot{u}=A u, & u \in \mathbb{R}^{m}, \\
\dot{v}=B v, & v \in \mathbb{R}^{n},
\end{array}
$$

Received by the editors December 18, 1996 and, in revised form, June 5, 1998.

2000 Mathematics Subject Classification. Primary 37D30, 37L45; Secondary 53C12, 37D10, $37 \mathrm{~K} 55$.

The first author was partially supported by NSF grant DMS-9622785 and the Isaac Newton Institute.

The second author was partially supported by NSF grant DMS-9622853.

The third author was partially supported by the Isaac Newton Institute.

(C)2000 American Mathematical Society 
with $\operatorname{Re} \sigma(A)>\gamma>\operatorname{Re} \sigma(B)$, where $A$ and $B$ are matrices, $\sigma(A)$ and $\sigma(B)$ are the spectra of $A$ and $B$, with Re denoting the real parts and $\gamma \in \mathbb{R}$ is a constant. For a given $u_{0} \in \mathbb{R}^{m}$, after $t>0$ the $n$-dimensional submanifold

$$
W_{0}=\left\{(u, v): u=u_{0}, v \in \mathbb{R}^{n}\right\}
$$

is carried by the flow to a new submanifold

$$
W_{t}=\left\{(u, v): u=e^{A t} u_{0}, v \in \mathbb{R}^{n}\right\} .
$$

Moreover, if both $\left(u_{1}, v_{1}\right)$ and $\left(u_{2}, v_{2}\right)$ lie in $W_{0}$ (i.e. $\left.u_{1}=u_{2}=u_{0}\right)$, then

$$
\left\|\left(e^{A t} u_{1}, e^{B t} v_{1}\right)-\left(e^{A t} u_{2}, e^{B t} v_{2}\right)\right\|=O\left(e^{\gamma t}\right), \quad \text { as } t \rightarrow+\infty,
$$

while a trajectory starting at a point not in $W_{0}$ departs from a trajectory starting in $W_{0}$ more rapidly than $C e^{\gamma t}$, as $t \rightarrow+\infty$. Thus, we are able to group points in $\mathbb{R}^{m+n}$ into equivalence classes according to their asymptotic behavior as $t \rightarrow+\infty$, and in this case each asymptotic class is a submanifold $u=$ constant. These submanifolds form a pseudo-stable foliation of $\mathbb{R}^{m+n}$. In general, the foliation will not consist of affine subspaces but can be thought of as perturbations of this case.

Invariant foliations with invariant manifolds have become a fundamental tool to study the qualitative properties of a flow or semiflow nearby invariant sets. They are extremely useful in that they can be used to track the asymptotic behavior of solutions and to provide coordinates in which systems of differential equations may be decoupled and normal forms derived.

In the study of the dynamics near an equilibrium or a periodic orbit, invariant manifolds and foliations serve as a convenient setting to describe the qualitative behavior of the local flows. In many cases they are useful tools for technical estimates which facilitate the study of the local bifurcation diagram (see, for example, [CLi]). Several other important concepts in dynamical systems are closely related to invariant manifolds and foliations. In finite dimensional space, the relations among invariant manifolds, invariant foliations, the $\lambda$-lemma, linearization and homoclinic bifurcation have been studied in D1. In BDL, invariant foliations are used to produce smooth conjugacy of flows on different center manifolds. Kirchgraber and Palmer $[\mathrm{KP}$ have recently given detailed results on invariant foliations and their applications to $C^{0}$ linearizations for finite dimensional systems. Very recently, de la Llave [L1] studied nonresonant invariant foliations for diffeomorphisms.

In [HPS] and [F1, [F2] and [F3], invariant foliations of the stable and unstable manifolds of a normally hyperbolic invariant manifold were obtained and some of their uses demonstrated. Since then, the applications of this theory to problems from science and engineering have flourished, especially, applications to singular perturbation problems, (see, for example, D2, [G], GS, [HW], [Jo, JK], [KW], [Li], $\mathrm{Sz}$ and $[\mathrm{Te}])$. Recently, Jones [Jo has given a clear discussion of the use of Fenichel's theorems as they apply to singular perturbation problems. He includes proofs of these theorems and important extensions of the $\lambda$-lemma (see also [JK]).

In the infinite dimensional setting, Li, McLaughlin, Shatah and Wiggins LMSW] obtained invariant foliations of center-stable and center-unstable manifolds of perturbations of a circle of stationary solutions for a nonlinear Schrödinger equation. These foliations were used in tracking trajectories and completing a shooting argument to discover homoclinic orbits. In [LW], Li and Wiggins obtained invariant foliations of overflowing manifolds for a $C^{r}(r \geq 2)$ group $S^{t}$ in a Hilbert space. They did this by using the method of Hadamard's graph transform. They also 
applied these results to the nonlinear Schrödinger equation to recover the results of [MMSW.

Ruelle $[\mathrm{Ru}]$ proved a result giving invariant stable and unstable fibrations almost everywhere on a compact invariant set for a semiflow in a Hilbert space. It was assumed that the linearized time- $t$ map is compact and injective with dense range. The results are therefore applicable to some parabolic PDE's. Mañé [Mn] extended Ruelle's results to semiflows in Banach space.

Considering the semiflow generated by a parabolic equation, Lu Lu1] constructed infinitely many invariant manifolds as perturbations of eigenspaces of the operator obtained by linearizing at an equilibrium point. With these and corresponding invariant foliations, a new coordinate system was constructed in a neighborhood of the equilibrium point. This facilitated a proof of a Hartman-Grobman theorem for scalar parabolic PDE's, which yields that the flow near a hyperbolic equilibrium point is structurally stable. In $[\mathrm{BL}$ a more general theorem on the existence of invariant foliations was proved. This theorem was then used to obtain a Hartman-Grobman result for both the phase-field system and the CahnHilliard equation. In Lu2], a Hartman-Grobman theorem for periodic orbits of time-periodic scalar parabolic PDE's is obtained by using invariant manifolds, invariant foliations and the Floquet theory obtained in [CLM]. The previously mentioned papers are concerned with dynamics in a neighborhood of an equilibrium or periodic orbit but Chow, Lin and Lu CLL proved a general result giving a stable fiber at each point of an inertial manifold, thereby giving a more global invariant foliation of infinite dimensional space.

Recently, Aulbach and Garay $\mathrm{AG}$ used invariant foliations to study partial linearization for noninvertible mappings near fixed points. Chen, Hale and Tan CHT considered a $C^{1}$ semiflow, in a Banach space $X$, with the linear part having an invariant splitting of $\mathrm{X}$ into pseudo-stable and pseudo-unstable invariant subspaces. This allowed them to prove the existence of a $C^{1}$ pseudo-unstable invariant manifold with a $C^{1}$ invariant foliation of $X$ based on the manifold, thus giving some extension of the results in CLL.

In this paper we start with a normally hyperbolic compact invariant manifold, $M$, for a semiflow and construct invariant foliations of the stable and the unstable manifold of $M$. What makes our analysis difficult is that we not only lack an inverse for the time- $t$ map but we also lack a Cartesian coordinate system around our invariant manifold since the normal bundle is not assumed to be trivial.

We organize this paper as follows: in Section 2 we state our main results; in Section 3 we collect some basic lemmas from [BLZ1]; in Section 4, we construct Lipschitz stable foliations and study their basic properties; in Section 5 we prove the smoothness of the stable foliations; in Section 6, we describe how, with appropriate modifications to our methods, the results of the previous sections carry over to unstable foliations.

\section{MAIn RESULtS}

Let $X$ be a Banach space with norm $|\cdot|$ and let $T^{t}$ be a $C^{1}$ semiflow defined on $X$; that is, it is continuous on $[0, \infty) \times X$, and for each $t \geq 0, T^{t}: X \rightarrow X$ is $C^{1}$, and $T^{t} \circ T^{s}(x)=T^{t+s}(x)$ for all $t, s \geq 0$ and $x \in X$.

Let $M \subset X$ be a $C^{2}$ compact connected invariant manifold, i.e., $T^{t}(M) \subset M$ for each $t \geq 0$. $M$ is said to be normally hyperbolic if the tangent bundle of $X$ 
restricted to $M$ splits into three continuous subbundles

$$
\left.T X\right|_{M}=X^{c} \oplus X^{u} \oplus X^{s},
$$

where $X^{c}$ is the tangent bundle of $M$, such that

(i) This splitting is invariant under the linearized semiflow, $D T^{t}$.

(ii) $\left.D T^{t}\right|_{X^{u}}$ expands and does so to a greater degree than does $\left.D T^{t}\right|_{X^{c}}$ while $\left.D T^{t}\right|_{X^{s}}$ contracts and does so to a greater degree than does $\left.D T^{t}\right|_{X^{c}}$.

The superscripts $c, u$ and $s$ stand for "center," "unstable," and "stable."

To be more specific, let $X_{m}^{c}, X_{m}^{u}$, and $X_{m}^{s}$ denote the fibers of the vector bundles $X^{c}, X^{u}$ and $X^{s}$ at $m \in M$, respectively. To say that the splitting is invariant under the linearized semiflow we mean

(H1) For each $m \in M$ and $t \geq 0$, if $m_{1}=T^{t}(m)$, then

$$
\left.D T^{t}(m)\right|_{X_{m}^{\alpha}}: X_{m}^{\alpha} \rightarrow X_{m_{1}}^{\alpha} \quad \text { for } \alpha=c, u, s
$$

and $\left.D T^{t}(m)\right|_{X_{m}^{u}}$ is an isomorphism from $X_{m}^{u}$ onto $X_{m_{1}}^{u}$.

The expanding and contracting condition (ii) means

(H2) There exists $t_{0} \geq 0$ and $\lambda<1$ such that for all $t \geq t_{0}$ and $m \in M$,

$$
\begin{aligned}
& \lambda \inf \left\{\left|D T^{t}(m) x^{u}\right|: x^{u} \in X_{m}^{u},\left|x^{u}\right|=1\right\}>\max \left\{1,\left\|\left.D T^{t}(m)\right|_{X_{m}^{c}}\right\|\right\}, \\
& \lambda \min \left\{1, \inf \left\{\left|D T^{t}(m) x^{c}\right|: x^{c} \in X_{m}^{c},\left|x^{c}\right|=1\right\}\right\}>\left\|\left.D T^{t}(m)\right|_{X_{m}^{s}}\right\|,
\end{aligned}
$$

where $\|\cdot\|$ is the usual linear operator norm.

Thus, fibers $X_{m}^{c}, X_{m}^{u}$ and $X_{m}^{s}$ are distinguished by the growth and decay rates of the flow, much as one sees with an exponential trichotomy.

We define the direct sum of bundles in the usual way, e.g.,

$$
X^{u} \oplus X^{s} \equiv\left\{\left(m, x^{u}+x^{s}\right): x^{u} \in X_{m}^{u} \text { and } x^{s} \in X_{m}^{s}, m \in M\right\}
$$

For $\epsilon>0$ we shall use the notation

$$
X^{\alpha}(\epsilon) \equiv\left\{\left(m, x^{\alpha}\right): x^{\alpha} \in X_{m}^{\alpha}, m \in M,\left|x^{\alpha}\right|<\epsilon\right\}, \quad \alpha=c, u, \text { and } s,
$$

and

$$
X^{u}(\epsilon) \oplus X^{s}(\epsilon) \equiv\left\{\left(m, x^{u}+x^{s}\right): x^{u} \in X_{m}^{u}, x^{s} \in X_{m}^{s}, m \in M,\left|x^{u}\right|<\epsilon,\left|x^{s}\right|<\epsilon\right\},
$$

the latter of which may be identified with a tubular neighborhood of $M$. The mapping which does this is $\Theta: X^{u} \oplus X^{s} \rightarrow X$ defined by $\Theta\left(m, x^{u}+x^{s}\right)=m+x^{u}+x^{s}$. For $m \in M$ we shall also use projections, $\Pi_{m}^{\alpha}$, associated with the decomposition of $X=X_{m}^{c} \oplus X_{m}^{u} \oplus X_{m}^{s}$, for $\alpha=c, u$, and $s$. We assume

(H3) The mapping from $M \subset X \rightarrow \mathcal{L}(X)$, the continuous linear operators on $X$, defined by $m \rightarrow \Pi_{m}^{\alpha}$ is $C^{1}$ for $\alpha=c, u$, and $s$.

Remark. If $|\cdot| X_{X^{\alpha}}$ is defined by $|(m, x)|_{X^{\alpha}}=|x|$ with $|\cdot|$ the norm in $X$, then the continuity in $m$ of the above mapping gives the bundle $X^{\alpha}$ a so-called Finsler structure.

In a forthcoming book BLZ2], we remove the assumption on the smoothness of the bundles; that is, (H3) can be removed and the assumption that the manifold $M$ is $C^{2}$ can be relaxed to require only $C^{1}$. The technical argument needed for this generalization is lengthy and does little to illuminate the main result, therefore, it is not included here.

We first quote a lemma from BLZ1] (Lemma 4.3) 
Lemma 2.1. For small $\epsilon>0, \Theta\left(X^{u}(\epsilon) \oplus X^{s}(\epsilon)\right)$ is a tubular neighborhood of $M$.

We summarize some of the main results obtained in BLZ1, which are needed in this paper, and state them as the following:

Theorem A. Let $t_{1}>t_{0}$. For each small $\epsilon>0, T^{t}$ has a unique $C^{1}$ center-stable manifold $W^{c s}(\epsilon)$ and a center-unstable manifold $W^{c u}(\epsilon)$ in the tubular neighborhood $\Theta\left(X^{u}(\epsilon) \oplus X^{s}(\epsilon)\right)$. These have the following properties:

(1) $M=W^{c s}(\epsilon) \cap W^{c u}(\epsilon)$. For each $m \in M$, the tangent spaces at $m$ are

$$
T_{m} W^{c s}(\epsilon)=X_{m}^{s} \oplus X_{m}^{c} \text { and } T_{m} W^{c u}(\epsilon)=X_{m}^{u} \oplus X_{m}^{c}
$$

(2) $T^{t} W^{c s}(\epsilon) \cap \Theta\left(X^{u}(\epsilon) \oplus X^{s}(\epsilon)\right) \subset W^{c s}(\epsilon)$. $T^{t} W^{c s}(\epsilon)$ converges to $M$ as $t \rightarrow+\infty$, and

$$
\begin{array}{r}
W^{c s}(\epsilon)=\left\{x \in \Theta\left(X^{u}(\epsilon) \oplus X^{s}(\epsilon)\right): T^{k t_{1}} x \in \Theta\left(X^{u}(\epsilon) \oplus X^{s}(\epsilon)\right)\right. \\
\text { for all } k>0\}
\end{array}
$$

(3) $T^{t_{1}}\left(W^{c s}(\epsilon)\right) \subset W^{c s}(\epsilon)$;

(4) $T^{t_{1}}: W^{c u}(\epsilon) \cap\left(T^{t_{1}}\right)^{-1}\left(W^{c u}(\epsilon)\right) \rightarrow W^{c u}(\epsilon)$ is a diffeomorphism. If we define $T^{-t}$ on $W^{c u}(\epsilon)$ in this way, then $T^{-t} W^{c u}(\epsilon)$ converges to $M$ as $t \rightarrow \infty$ and $W^{c u}(\epsilon)=\left\{x \in \Theta\left(X^{u}(\epsilon) \oplus X^{s}(\epsilon)\right):\right.$ for all $k>0$, there exists

$$
\left.y_{k} \in \Theta\left(X^{u}(\epsilon) \oplus X^{s}(\epsilon)\right) \text { satisfying } T^{k t_{1}} y_{k}=x\right\} \text {. }
$$

Remark. (2) implies that $W^{c s}(\epsilon)$ consists of all points in the tubular neighborhood whose forward orbits stay in the tubular neighborhood and approach $M$ and (4) implies that $W^{c u}(\epsilon)$ consists of all points in the tubular neighborhood whose backward orbits exist and stay in the tubular neighborhood and approach $M$, where the backward orbits are defined by the preimages.

The proof of the existence of the two invariant manifolds is different. To obtain $W^{c u}$, we apply $T^{t}$ to Lipschitz graphs over the bundle $X^{u}$ and, essentially, find it is a contraction on the space of Lipschitz graphs. To obtain $W^{c s}$, the natural inclination is to reverse time and apply the preceeding argument. This is not possible, however, since we only have a semiflow. Normal hyperbolicity allows us to overcome this difficulty and we can show that for any Lipschitz graph, $g$, over $X^{s}$, there is a Lipschitz graph $\tilde{g}$ such that $T^{t}$ maps $\tilde{g}$ into $g$. This mapping, which assigns $\tilde{g}$ to any given $g$, is a contraction whose fixed point is $W^{c s}$.

Note that $W^{c s}(\epsilon)$ and $W^{c u}(\epsilon)$ are invariant manifolds for the map $T^{t_{1}}$ and also for the semiflow $T^{t}$ in some sense. The properties above give some information about the asymptotic behavior of the semiflow $T^{t}$ in the tubular neighborhood of $M$. The behavior on $W^{c s}(\epsilon)$ and $W^{c u}(\epsilon)$ is particularly important. On $W^{c s}(\epsilon)$, the motion is basically along two directions: The center direction and the stable direction. Along the stable direction the semiflow just decays exponentially. Thus, the motion along the center direction determines the asymptotic behavior. For each $x \in W^{c s}(\epsilon)$, we shall find a unique $m \in M$ so that $T^{t}(x)$ and $T^{t}(m)$ have the same asymptotic behavior at an exponential rate. In this way, $W^{c s}(\epsilon)$ can be decomposed as the disjoint union of submanifolds according to the asymptotic behavior; these manifolds being indexed by points of $M$. Similar statements hold for $W^{c u}(\epsilon)$. The following are the main results of this paper: 
Theorem 2.2. For small $\epsilon>0$ there exists a unique family of $C^{1}$ submanifolds $\left\{W_{m}^{s s}(\epsilon): m \in M\right\}$ of $W^{c s}(\epsilon)$ satisfying:

(1) For each $m \in M, M \cap W_{m}^{s s}(\epsilon)=\{m\}$, the tangent space $T_{m} W_{m}^{s s}(\epsilon)=X_{m}^{s}$ and $W_{m}^{s s}(\epsilon)$ varies continuously with respect to $m$ on $M$.

(2) If $m_{1}, m_{2} \in M, m_{1} \neq m_{2}$, then $W_{m_{1}}^{s s}(\epsilon) \cap W_{m_{2}}^{s s}(\epsilon)=\emptyset$ and $W^{c s}(\epsilon)=$ $\bigcup_{m \in M} W_{m}^{s s}(\epsilon)$.

(3) For $m \in M, T^{t_{1}}\left(W_{m}^{s s}(\epsilon)\right) \subset W_{T^{t_{1}} m}^{s s}(\epsilon)$.

(4) For all $m \in M$ and $t>0, T^{t}\left(W_{m}^{s s}(\epsilon)\right) \cap \Theta\left(X^{u}(\epsilon) \oplus X^{s}(\epsilon)\right) \subset W_{T^{t}(m)}^{s s}(\epsilon)$.

(5) For $x \in W_{m}^{s s}(\epsilon)$ and $m \neq m_{1} \in M$, we have $\frac{\left|T^{t}(x)-T^{t}(m)\right|}{\left|T^{t}(x)-T^{t}\left(m_{1}\right)\right|} \rightarrow 0$ exponentially as $t \rightarrow+\infty$.

(6) For $x, y \in W_{m}^{s s}(\epsilon),\left|T^{t}(x)-T^{t}(y)\right| \rightarrow 0$ exponentially as $t \rightarrow+\infty$.

For the invariant foliation of $W^{c u}$ into unstable fibers, we have

Theorem 2.3. For small $\epsilon>0$ there exists a family of $C^{1}$ submanifolds $\left\{W_{m}^{u u}(\epsilon)\right.$ : $m \in M\}$ of $W^{c u}(\epsilon)$ satisfying:

(1) For each $m \in M, M \cap W_{m}^{u u}(\epsilon)=\{m\}, T_{m} W_{m}^{u u}(\epsilon)=X_{m}^{u}$ and $W_{m}^{u u}(\epsilon)$ varies continuously with respect to $m$ on $M$.

(2) If $m_{1}, m_{2} \in M, m_{1} \neq m_{2}$, then $W_{m_{1}}^{u u}(\epsilon) \cap W_{m_{2}}^{u u}(\epsilon)=\emptyset$ and $W^{c u}(\epsilon)=$ $\bigcup_{m \in M} W_{m}^{u u}(\epsilon)$.

(3) For all $m \in M, T^{t_{1}}: W_{m}^{u u}(\epsilon) \cap\left(T^{t_{1}}\right)^{-1}\left(W_{T^{t_{1}(m)}}^{u u}(\epsilon)\right) \rightarrow W_{T^{t_{1}(m)}}^{u u}(\epsilon)$ is a diffeomorphism.

(4) For $x \in W_{m}^{u u}(\epsilon)$, if $T^{t}(x) \in \Theta\left(X^{u}(\epsilon) \oplus X^{s}(\epsilon)\right)$ for all $0<t<t_{2}$, for some $t_{2}$, then $T^{t}(x) \in W_{T^{t}(m)}^{u u}(\epsilon)$ for all $0<t<t_{2}$.

(5) For $x \in W_{m}^{u u}(\epsilon)$ and $m \neq m_{1} \in M$, we have $\frac{\left|T^{-t}(x)-T^{-t}(m)\right|}{\left|T^{-t}(x)-T^{-t}\left(m_{1}\right)\right|} \rightarrow 0$ exponentially as $t \rightarrow+\infty$.

(6) For $x, y \in W_{m}^{u u}(\epsilon)$, we have $\left|T^{-t}(x)-T^{-t}(y)\right| \rightarrow 0$ exponentially as $t \rightarrow$ $+\infty$.

We shall call each submanifold $W_{m}^{s s}(\epsilon)$ a stable fiber and $W_{m}^{u u}(\epsilon)$ an unstable fiber. The stable foliation is the decomposition of $W^{c s}(\epsilon)$ into the disjoint union of the stable fibers, and similarly the unstable foliation is the decomposition of $W^{c u}(\epsilon)$ into the disjoint union of the unstable fibers.

\section{BASIC LEMMAS}

In this section we review some basic lemmas including a lemma on cone invariance and results relating three coordinate systems which were introduced in BLZ1.

The following lemma summarizes Lemmas 4.1, 4.2, and 4.3 from [BLZ1], which give fundamental properties of the vector bundles $X^{\alpha}$ for $\alpha=u, s, c$.

Lemma 3.1. The following statements hold:

(i) For $m_{1}, m_{2} \in M, X_{m_{1}}^{\alpha}$ is isomorphic to $X_{m_{2}}^{\alpha}$ for $\alpha=u, s, c$;

(ii) $X^{\alpha}$ for $\alpha=u, s, c$, is a $C^{1}$ bundle;

(iii) There exists $\epsilon>0$ such that $\Theta$ is a $C^{1}$ diffeomorphism from $X^{u}(\epsilon) \oplus X^{s}(\epsilon)$ onto a neighborhood of $M$.

We recall the concept of an $\eta$-neighborhood of a point $m_{0} \in M$.

Definition. For $m_{0} \in M$ and $\eta \in(0,1)$, a neighborhood $U$ of $m_{0}$ in $X$ is said to be an $\eta$-neighborhood if $\left\|\Pi_{m_{1}}^{\alpha}-\Pi_{m_{2}}^{\alpha}\right\| \leq \eta$ for all $m_{1}, m_{2} \in U \cap M$, for $\alpha=c, u$, and $s$. 
Let $\eta<\sqrt{2}-1$ and $\bar{m} \in V_{m} \cap M$, where $V_{m}$ is an $\eta$-neighborhood of $m$, then we have the following estimates for the projections

$$
\left|\Pi_{\bar{m}}^{\alpha} x\right| \geq(1-\eta)|x|,
$$

for $x \in X_{m}^{\alpha}$ and

$$
\left\|\left.\Pi_{\bar{m}}^{\alpha}\right|_{X_{m}^{\alpha}}\right\| \leq 1+\eta \text { and }\left\|\left(\left.\Pi_{\bar{m}}^{\alpha}\right|_{X_{m}^{\alpha}}\right)^{-1}\right\| \leq \frac{1}{1-\eta} .
$$

Let $\epsilon_{1}>0$ be sufficiently small. For $m_{0} \in M$, let $B\left(m_{0}, r\right)$ be the ball centered at $m_{0}$ of radius $r$, where $r$ is chosen so that this is an $\eta$-neighborhood with $\eta<\sqrt{2}-1$. In fact, by compactness of $M, r$ can be chosen to be independent of $m_{0}$. Next we recall three coordinate systems introduced in BLZ1]. We express each point in a tubular neighborhood in terms of three coordinate systems as follows. For each point $x \in \Theta\left(X^{u}(\epsilon) \oplus X^{s}(\epsilon)\right)$, Lemma 3.1 (iii) gives the first coordinates $\left(m, x^{u}+x^{s}\right)$ $\in X^{u}\left(\epsilon_{1}\right) \oplus X^{s}\left(\epsilon_{1}\right)$, where $x=m+x^{u}+x^{s}$. The advantage of this coordinate system is that it is global in a neighborhood of $M$, but the disadvantage is that the coordinate spaces vary with the base point $m$. For $m_{0} \in M$ such that $m \in B\left(m_{0}, r\right)$, since $X=X_{m_{0}}^{u} \oplus X_{m_{0}}^{s} \oplus X_{m_{0}}^{c}$, there is a unique $\bar{x}^{u}+\bar{x}^{s}+\bar{x}^{c} \in X_{m_{0}}^{u} \oplus X_{m_{0}}^{s} \oplus X_{m_{0}}^{c}$ such that

$$
m+x^{u}+x^{s}=m_{0}+\bar{x}^{u}+\bar{x}^{s}+\bar{x}^{c},
$$

giving a Cartesian coordinate system in a neighborhood of $m_{0}$. The advantage of this system is that the coordinate spaces do not depend on the base point $m$, which plays a crucial role in proving the existence and smoothness of invariant foliations. If we trivialize the bundle near $m_{0}$, we have the third coordinate system modified from the first one. From (3.2) we find there exists a unique $\tilde{x}^{u}+\tilde{x}^{s} \in X_{m_{0}}^{u} \oplus X_{m_{0}}^{s}$ such that

$$
m+x^{u}+x^{s}=m+\Pi_{m}^{u} \tilde{x}^{u}+\Pi_{m}^{s} \tilde{x}^{s} .
$$

This defines the third coordinate system $\left(m, \tilde{x}^{u}, \tilde{x}^{s}\right)$.

We now consider the relationships between these coordinate systems. We still denote the ball with center 0 and radius $\epsilon$ in $X_{m}^{\alpha}$ by $X_{m}^{\alpha}(\epsilon)$. Take two points $\left(m_{i}, x_{i}^{u}+x_{i}^{s}\right) \in X_{m_{i}}^{u}\left(\epsilon_{1}\right) \oplus X_{m_{i}}^{s}\left(\epsilon_{1}\right)$ with $m_{i} \in B\left(m_{0}, r\right) \cap M, i=1,2$. Then we have the three representations

$$
\begin{aligned}
& m_{i}+x_{i}^{u}+x_{i}^{s} \\
& =m_{0}+\bar{x}_{i}^{u}+\bar{x}_{i}^{s}+\bar{x}_{i}^{c} \\
& =m_{i}+\Pi_{m_{i}}^{u} \tilde{x}_{i}^{u}+\Pi_{m_{i}}^{s} \tilde{x}^{s},
\end{aligned}
$$

where $\bar{x}_{i}^{\alpha} \in X_{m_{0}}^{\alpha}, \alpha=u, s, c, \tilde{x}_{i}^{\alpha} \in X_{m_{0}}^{\alpha}, \alpha=u, s$.

The next result, which is Lemma 4.5 in [BLZ1, estimates how the coordinates differ from each other.

\section{Lemma 3.2.}

$$
\begin{aligned}
& \left|\bar{x}_{1}^{c}-\bar{x}_{2}^{c}-\left(m_{1}-m_{2}\right)\right| \\
& \leq C \epsilon_{1}\left|m_{1}-m_{2}\right|+C\left|m_{i}-m_{0}\right|\left(\left|\tilde{x}_{1}^{u}-\tilde{x}_{2}^{u}\right|+\left|\tilde{x}_{1}^{s}-\tilde{x}_{2}^{s}\right|\right)
\end{aligned}
$$

and

$$
\begin{aligned}
& \left|\bar{x}_{1}^{\alpha}-\bar{x}_{2}^{\alpha}-\left(\tilde{x}_{1}^{\alpha}-\tilde{x}_{2}^{\alpha}\right)\right| \\
& \leq C \epsilon_{1}\left|m_{1}-m_{2}\right|+C\left|m_{i}-m_{0}\right|\left(\left|\tilde{x}_{1}^{u}-\tilde{x}_{2}^{u}\right|+\left|\tilde{x}_{1}^{s}-\tilde{x}_{2}^{s}\right|\right)
\end{aligned}
$$


for $i=1,2, \alpha=u, s$, where $C$ is a positive constant which depends only on the projections.

In the remainder of this paper, we shall use $C$ as a generic constant depending only on the projections and the time- $t_{1}$ map $T^{t_{1}}$ for a fixed $t_{1}>t_{0}$.

We shall confine our study to the tubular neighborhood $\Theta\left(X^{u}(\epsilon) \oplus X^{s}(\epsilon)\right)$ of $M$. For $\left(m_{0}, x_{0}^{u}+x_{0}^{s}\right) \in X^{u}(\epsilon) \oplus X^{s}(\epsilon)$, and for $\mu>0$, define the cones and truncated cones

$$
\begin{aligned}
& K_{u}\left(m_{0}+x_{0}^{u}+x_{0}^{s}, \mu\right)=\{ m_{0}+x_{0}^{u}+x_{0}^{s}+x^{u}+x^{s}+x^{c}: \\
&\left.x^{\alpha} \in X_{m_{0}}^{\alpha} \text { for } \alpha=u, s, \text { and } c, \mu\left|x^{u}\right|>\left(\left|x^{c}\right|+\left|x^{s}\right|\right)\right\}, \\
& K_{u}\left(m_{0}+x_{0}^{u}+x_{0}^{s}, \mu, \epsilon\right)=\{ m_{0}+x_{0}^{u}+x_{0}^{s}+x^{u}+x^{s}+x^{c} \\
& \in\left.K_{u}\left(m_{0}+x_{0}^{u}+x_{0}^{s}, \mu\right):\left|x^{\alpha}\right|<\epsilon \text { for } \alpha=u, s, \text { and } c\right\}, \\
& K_{c u}\left(m_{0}+x_{0}^{u}+x_{0}^{s}, \mu\right)=\{ m_{0}+x_{0}^{u}+x_{0}^{s}+x^{u}+x^{s}+x^{c}: \\
&\left.x^{\alpha} \in X_{m_{0}}^{\alpha} \text { for } \alpha=u, s, \text { and } c, \mu\left(\left|x^{u}\right|+\left|x^{c}\right|\right)>\left|x^{s}\right|\right\}, \\
& K_{c u}\left(m_{0}+x_{0}^{u}+x_{0}^{s}, \mu, \epsilon\right)=\{ m_{0}+x_{0}^{u}+x_{0}^{s}+x^{u}+x^{s}+x^{c} \\
&\left.\in K_{c u}\left(m_{0}+x_{0}^{u}+x_{0}^{s}, \mu\right):\left|x^{\alpha}\right|<\epsilon \text { for } \alpha=u, s, \text { and } c\right\} .
\end{aligned}
$$

Then we have the following cone invariance, which follows from Lemma 5.5 in BLZ1.

Lemma 3.3. For any $\lambda_{1} \in(\lambda, 1)$ and $\mu>0$, there exists $\epsilon^{*}$ such that if $\epsilon<\epsilon^{*}$, then for any $x \in \Theta\left(X^{u}(\epsilon) \oplus X^{s}(\epsilon)\right)$ with $T^{t_{1}}(x) \in \Theta\left(X^{u}(\epsilon) \oplus X^{s}(\epsilon)\right)$ we have

$$
T^{t_{1}} K_{u}(x, \mu, \epsilon) \subset K_{u}\left(T^{t_{1}}(x), \lambda_{1} \mu\right)
$$

and

$$
T^{t_{1}} K_{c u}(x, \mu, \epsilon) \subset K_{c u}\left(T^{t_{1}}(x), \lambda_{1} \mu\right) .
$$

The compactness of $M$ allows us to establish the following, found as Lemma 2.1 in BLZ1].

Lemma 3.4. For any $t>0, T^{t}$ is a diffeomorphism from $M$ to $M$.

The next lemma follows from the normal hyperbolicity condition. It appears as Lemma 8.2 in BLZ1.

Lemma 3.5. There exists $\epsilon^{*}>0$ such that for $\epsilon<\epsilon^{*}$ if $x=m+x^{u}+x^{s} \epsilon$ $\Theta\left(X^{u}(\epsilon) \oplus X^{s}(\epsilon)\right)$, then the following estimates hold:

(i) $\left\|\left(\left.\Pi_{\tilde{m}}^{u} D T^{t_{1}}(x)\right|_{X_{m}^{u}}\right)^{-1}\right\|<\lambda$,

(ii) $\left\|\left(\left.\Pi_{\tilde{m}}^{u} D T^{t_{1}}(x)\right|_{X_{m}^{u}} ^{u}\right)^{-1}\right\|\left\|\left.\Pi_{\tilde{m}}^{c} D T^{t_{1}}(x)\right|_{X_{m}^{c}}\right\|<\lambda$, 
(iii) $\left\|\left(\left.\Pi_{\tilde{m}}^{c} D T^{t_{1}}(x)\right|_{X_{m}^{c}}\right)^{-1}\right\|\left\|\left.\Pi_{\tilde{m}}^{s} D T^{t_{1}}(x)\right|_{X_{m}^{s}}\right\|<\lambda$, where $\tilde{m}$ is given by $T^{t_{1}}(x)=\tilde{m}+\tilde{x}^{u}+\tilde{x}^{s}$.

Let $\epsilon_{0} \in(0, r / 2)$ be fixed such that $\Theta\left(X^{u}\left(\epsilon_{0}\right) \oplus X^{s}\left(\epsilon_{0}\right)\right)$ is a tubular neighborhood of $M$. From Lemma 5.4 in BLZ1] we have

Lemma 3.6. For $\epsilon_{1} \in\left(0, \epsilon_{0}\right)$, there exists a positive constant $\epsilon^{*}$ such that if $\epsilon<$ $\epsilon^{*}$, then for all $\left(m, x^{u}+x^{s}\right) \in \overline{X^{u}(\epsilon)} \oplus \overline{X^{s}(\epsilon)}$ (the bar means closure) and $\hat{x}^{\alpha} \in$ $\overline{X_{m}^{\alpha}(3 \epsilon)}, \alpha=u, s, c$,

$$
m+x^{u}+x^{s}+\hat{x}^{u}+\hat{x}^{s}+\hat{x}^{c} \in \Theta\left(X^{u}\left(\epsilon_{1}\right) \oplus X^{s}\left(\epsilon_{1}\right)\right)
$$

and

$$
T^{t_{1}}\left(m+x^{u}+x^{s}+\hat{x}^{u}+\hat{x}^{s}+\hat{x}^{c}\right) \in \Theta\left(X^{u}\left(\epsilon_{1}\right) \oplus X^{s}\left(\epsilon_{1}\right)\right) .
$$

\section{EXISTENCE OF LipsCHitZ INVARIANT STABLE FOLIATION}

In this section, we establish the existence of an invariant foliation (we call it a stable foliation) of $W^{c s}(\epsilon)$ for the time- $t_{1}$ map $T^{t_{1}}$. Later, we prove that this stable foliation is invariant for the semiflow $T^{t}$.

We first define a stable fiber through $x=m+x^{u}+x^{s} \in \Theta\left(X^{u}(\epsilon) \oplus X^{s}(\epsilon)\right)$. Consider a map associated with $x$

$$
f_{x}: X_{m}^{s}(3 \epsilon) \rightarrow X_{m}^{u}(\epsilon) \oplus X_{m}^{c}(\epsilon) .
$$

Here, we choose $\epsilon>0$ small enough so that $\Theta\left(X^{u}(4 \epsilon) \oplus X^{s}(4 \epsilon)\right)$ is a tubular neighborhood of $M$.

Definition. $f_{x}: X_{m}^{s}(3 \epsilon) \rightarrow X_{m}^{u}(\epsilon) \oplus X_{m}^{c}(\epsilon)$ is called a stable fiber at $x$ of Lipschitz constant $\mu$ if

(i) $f_{x}(0)=0$,

(ii) $f_{x}(\cdot)$ is Lipschitz continuous with Lipschitz constant $\mu$; that is,

$$
\left|f\left(y_{1}^{s}\right)-f\left(y_{2}^{s}\right)\right| \leq \mu\left|y_{1}^{s}-y_{2}^{s}\right| \text { for } y_{1}^{s}, y_{2}^{s} \in X_{m}^{s}(3 \epsilon),
$$

where the norm in the direct sum of spaces is defined to be the sum of the norms. For brevity, we just call $f_{x}$ a stable fiber.

We denote the graph of $f_{x}$ by

$$
\operatorname{graph}\left(f_{x}\right)=\left\{x+y^{s}+f_{x}\left(y^{s}\right): y^{s} \in X_{m}^{s}(3 \epsilon)\right\} .
$$

In the remainder of this paper, we shall fix $\mu \in\left(0, \frac{1}{3}\right)$.

Next, we define a space of families of stable fibers in which we shall construct an invariant foliation of $W^{c s}(\epsilon)$. Define

$$
\begin{aligned}
\Gamma_{\epsilon}^{s s}=\left\{F=\left\{f_{x}\right\}_{x \in \overline{W^{c s}(\epsilon)}}:\right. & f_{x} \text { is a stable fiber and } \\
& \left.f_{x}\left(y^{s}\right) \text { is uniformly continuous in } x \in \overline{W^{c s}(\epsilon)}\right\} .
\end{aligned}
$$

We shall call $f_{x}$ the fiber (or leaf) of $F$ at $x$. The uniform continuity of the fibers $f_{x}$ in $x$ will be defined precisely, later. 
The main theorem in this section is

Theorem 4.1. There exists $\epsilon^{*}>0$ such that for $\epsilon<\epsilon^{*}, W^{c s}(\epsilon)$ has a unique invariant foliation given by

$$
W^{c s}(\epsilon)=\bigcup_{m \in M} W_{m}^{s s}(\epsilon)
$$

where $W_{m}^{s s}(\epsilon)=\operatorname{graph}\left(f_{m}\right) \cap W^{c s}(\epsilon)$ satisfying:

(1) $f_{m}$ is a stable fiber.

(2) $W_{m}^{s s}(\epsilon)$ is continuous in $m \in M$.

(3) $W_{m_{1}}^{s s}(\epsilon) \cap W_{m_{2}}^{s s}(\epsilon)=\varnothing$ for $m_{1}, m_{2} \in M, m_{1} \neq m_{2}$.

(4) For $m \in M, T^{t_{1}}\left(W_{m}^{s s}(\epsilon)\right) \subset W_{T^{t_{1}}(m)}^{s s}(\epsilon)$.

(5) For all $m \in M$ and $t>0, T^{t}\left(W_{m}^{s s}(\epsilon)\right) \cap \Theta\left(X^{u}(\epsilon) \oplus X^{s}(\epsilon)\right) \subset W_{T^{t}(m)}^{s s}(\epsilon)$.

(6) For $x \in W_{m}^{s s}(\epsilon)$ and $m \neq m_{1} \in M$, we have $\frac{\left|T^{t}(x)-T^{t}(m)\right|}{\left|T^{t}(x)-T^{t}\left(m_{1}\right)\right|} \rightarrow 0$ exponentially as $t \rightarrow+\infty$.

(7) For $x, y \in W_{m}^{s s}(\epsilon),\left|T^{t}(x)-T^{t}(y)\right| \rightarrow 0$ exponentially as $t \rightarrow+\infty$.

The proof of this theorem is based on the following propositions and lemmas.

Proposition 4.2. There exists $\epsilon^{*}>0$ such that for $0<\epsilon<\epsilon^{*}$ and any $F \in \Gamma_{\epsilon}^{s s}$, there exists a unique $\tilde{F} \in \Gamma_{\epsilon}^{s s}$ such that each fiber of $\tilde{F}$ is mapped into a fiber of $F$ by $T^{t_{1}}$; that is, given any $x_{1}=m_{1}+x_{1}^{u}+x_{1}^{s} \in W^{c s}(\epsilon)$ and any $y_{1}^{s} \in X_{m_{1}}^{s}(3 \epsilon)$, there exists a unique $y_{2}^{s} \in X_{m_{2}}^{s}(3 \epsilon)$, such that

$$
T^{t_{1}}\left(x_{1}+y_{1}^{s}+\tilde{f}_{x_{1}}\left(y_{1}^{s}\right)\right)=x_{2}+y_{2}^{s}+f_{x_{2}}\left(y_{2}^{s}\right)
$$

where $m_{2}$ and $x_{2}$ are given by $T^{t_{1}}\left(x_{1}\right)=x_{2}=m_{2}+x_{2}^{u}+x_{2}^{s}$.

This will be proved through a sequence of lemmas.

Throughout this section, for $x_{1}=m_{1}+x_{1}^{u}+x_{1}^{s} \in \Theta\left(X^{u}(\epsilon) \oplus X^{s}(\epsilon)\right)$, we denote $T^{t_{1}}\left(x_{1}\right)$ by $x_{2}$ and write $x_{2}=T^{t_{1}}\left(x_{1}\right)=m_{2}+x_{2}^{u}+x_{2}^{s}$.

Lemma 4.3. There exists $\epsilon^{*}>0$ such that if $0<\epsilon<\epsilon^{*}$, then for all $x_{1} \epsilon$ $\Theta\left(X^{u}(\epsilon) \oplus X^{s}(\epsilon)\right)$ with $T^{t_{1}}\left(x_{1}\right) \in \Theta\left(X^{u}(\epsilon) \oplus X^{s}(\epsilon)\right), y_{1}^{s} \in X_{m_{1}}^{s}(3 \epsilon)$ and stable fiber $f_{x_{2}}$, there exists a unique $y_{1}^{u}+y_{1}^{c} \in X_{m_{1}}^{u}(\epsilon) \oplus X_{m_{1}}^{c}(\epsilon)$ such that

$$
T^{t_{1}}\left(x_{1}+y_{1}^{s}+y_{1}^{u}+y_{1}^{c}\right) \in \operatorname{graph}\left(f_{x_{2}}\right) .
$$

Proof. For $x_{1}=m_{1}+x_{1}^{u}+x_{1}^{s} \in \Theta\left(X^{u}(\epsilon) \oplus X^{s}(\epsilon)\right)$ with $T^{t_{1}}\left(x_{1}\right) \in \Theta\left(X^{u}(\epsilon) \oplus X^{s}(\epsilon)\right)$, we write $x_{2}=T^{t_{1}}\left(x_{1}\right)$ as

$$
x_{2}=T^{t_{1}}\left(x_{1}\right)=m_{2}+x_{2}^{u}+x_{2}^{s},
$$

where $x_{2}^{\alpha} \in X_{m_{2}}^{\alpha}(\epsilon)$ for $\alpha=u, s$. For any $y^{u}+y^{c} \in \overline{X_{m_{1}}^{u}(\epsilon)} \oplus \overline{X_{m}^{c}(\epsilon)}$, we write

$$
T^{t}\left(x_{1}+y^{u}+y_{1}^{s}+y^{c}\right)=x_{2}+\bar{y}^{u}+\bar{y}^{s}+\bar{y}^{c}
$$

where $\bar{y}^{\alpha} \in X_{m_{2}}^{\alpha}$ for $\alpha=u, s, c$.

It is enough to prove that there is a unique $y^{u}+y^{c} \in X_{m_{1}}^{u}(\epsilon) \oplus X_{m_{1}}^{c}(\epsilon)$ such that $\bar{y}^{u}+\bar{y}^{c}=f_{x_{2}}\left(\bar{y}^{s}\right)$. To find such $y^{u}+y^{c}$, we consider a map $A=A^{u}+A^{c}$ from $X_{m_{1}}^{u}(\epsilon) \oplus X_{m_{1}}^{c}(\epsilon)$ to $X_{m_{1}}^{u} \oplus X_{m_{1}}^{c}$ defined by

$$
A^{u}\left(y^{u}+y^{c}\right)=\left(\left.\Pi_{m_{2}}^{u} D T^{t_{1}}\left(m_{1}\right)\right|_{X_{m_{1}}^{u}}\right)^{-1}\left(\tilde{y}^{u}-\bar{y}^{u}\right)+y^{u}
$$


and

$$
A^{c}\left(y^{u}+y^{c}\right)=\left(\left.\Pi_{m_{2}}^{c} D T^{t_{1}}\left(m_{1}\right)\right|_{X_{m_{1}}^{c}}\right)^{-1}\left(\tilde{y}^{c}-\bar{y}^{c}\right)+y^{c},
$$

where $\tilde{y}^{u}+\tilde{y}^{c}=f_{x_{2}}\left(\bar{y}^{s}\right)$.

We claim that $A$ is a contraction on $\overline{X_{m_{1}}^{u}(\epsilon)} \oplus \overline{X_{m_{1}}^{c}(\epsilon)}$.

We first prove that $A$ is well defined. Note that $D T^{t_{1}}\left(m_{1}\right): X_{m_{1}}^{u} \rightarrow X_{m_{1}}^{u}$ and $D T^{t_{1}}\left(m_{1}\right): X_{m_{1}}^{c} \rightarrow X_{\overline{m_{1}}}^{c}$ have bounded inverses from hypothesis (H2), where $\overline{m_{1}}=$ $T^{t_{1}}\left(m_{1}\right)$. From Lemma 3.5 , by choosing $\epsilon^{*}$ small enough, both $\left.\Pi_{m_{2}}^{u} D T^{t_{1}}\left(m_{1}\right)\right|_{X_{m_{1}}^{u}}$ and $\left.\Pi_{m_{2}}^{c} D T^{t_{1}}\left(m_{1}\right)\right|_{X_{m_{1}}^{c}}$ have bounded inverses.

Applying the projection $\Pi_{m_{2}}^{s}$ to (4.3) we have

$$
\bar{y}^{s}=\Pi_{m_{2}}^{s}\left(T^{t_{1}}\left(x_{1}+y^{u}+y_{1}^{s}+y^{c}\right)-x_{2}\right) .
$$

Using the Taylor expansion of $T^{t_{1}}\left(x_{1}+y^{u}+y_{1}^{s}+y^{c}\right)$ and hypothesis (H2), we obtain

$$
\begin{aligned}
\left|\bar{y}^{s}\right| & =\left|\Pi_{m_{2}}^{s}\left(T^{t_{1}}\left(m_{1}+x_{1}^{u}+x_{1}^{s}+y^{u}+y_{1}^{s}+y^{c}\right)-T^{t_{1}}\left(m_{1}+x_{1}^{u}+x_{1}^{s}\right)\right)\right| \\
& \leq\left|\Pi_{m_{2}}^{s} D T^{t_{1}}\left(m_{1}\right)\left(y^{u}+y_{1}^{s}+y^{c}\right)\right|+O(\epsilon)\left|y^{u}+y_{1}^{s}+y^{c}\right| \\
& \leq\left|\Pi_{\bar{m}_{1}}^{s} D T^{t_{1}}\left(m_{1}\right) y_{1}^{s}\right|+O(\epsilon)\left|y^{u}+y_{1}^{s}+y^{c}\right| \\
& \leq \lambda\left|y_{1}^{s}\right|+O(\epsilon)\left|y^{u}+y_{1}^{s}+y^{c}\right| \\
& \leq(3 \lambda+O(\epsilon)) \epsilon<3 \epsilon
\end{aligned}
$$

provided that $\epsilon^{*}$ is sufficiently small. Here $0<\lambda<1$ is used. Hence, $A$ is well defined.

Next we show that $A: \overline{X_{m_{1}}^{u}(\epsilon)} \oplus \overline{X_{m_{1}}^{c}(\epsilon)} \rightarrow X_{m_{1}}^{u} \oplus X_{m_{1}}^{c}$ is a contraction. For $y_{1}^{u}+y_{1}^{c}, y_{2}^{u}+y_{2}^{c} \in X_{m_{1}}^{u}(\epsilon) \oplus X_{m_{1}}^{c}(\epsilon)$. We write

$$
T^{t_{1}}\left(x_{1}+y_{i}^{u}+y_{1}^{s}+y_{i}^{c}\right)=x_{2}+\bar{y}_{i}^{u}+\bar{y}_{i}^{s}+\bar{y}_{i}^{c}
$$

and set

$$
\tilde{y}_{i}^{u}+\tilde{y}_{i}^{c}=f_{x_{2}}\left(\bar{y}_{i}^{s}\right)
$$

for $i=1,2$. Note that from (4.4), $\left|\bar{y}_{i}^{s}\right|<3 \epsilon$.

Let $\ell(\tau)=(1-\tau)\left(y_{1}^{u}+y_{1}^{c}\right)+\tau\left(y_{2}^{u}+y_{2}^{c}\right)+x_{1}+y_{1}^{s}$ for $0 \leq \tau \leq 1$.

For $\alpha=u, c$, compute

$$
\begin{aligned}
& \left(\left.\Pi_{m_{2}}^{\alpha} D T^{t_{1}}\left(m_{1}\right)\right|_{X_{m_{1}}^{\alpha}}\right)^{-1}\left(\bar{y}_{2}^{\alpha}-\bar{y}_{1}^{\alpha}\right) \\
& =\left(\left.\Pi_{m_{2}}^{\alpha} D T^{t_{1}}\left(m_{1}\right)\right|_{X_{m_{1}}^{\alpha}}\right)^{-1} \int_{0}^{1} \Pi_{m_{2}}^{\alpha} D T^{t_{1}}(\ell(\tau))\left(y_{2}^{u}-y_{1}^{u}+y_{2}^{c}-y_{1}^{c}\right) d \tau \\
& =\left(y_{2}^{\alpha}-y_{1}^{\alpha}\right)+O(\epsilon)\left(\left|y_{2}^{u}-y_{1}^{u}\right|+\left|y_{2}^{c}-y_{1}^{c}\right|\right) .
\end{aligned}
$$

In order to show that $A$ is a contraction, we also need to estimate

$$
\begin{aligned}
\left|\bar{y}_{2}^{s}-\bar{y}_{1}^{s}\right| & =\left|\int_{0}^{1} \Pi_{m_{2}}^{s} D T(\ell(\tau))\left(y_{2}^{u}-y_{1}^{u}+y_{2}^{c}-y_{1}^{c}\right) d \tau\right| \\
& \leq O(\epsilon)\left(\left|y_{2}^{u}-y_{1}^{u}\right|+\left|y_{2}^{c}-y_{1}^{c}\right|\right) .
\end{aligned}
$$

From (4.6), by using (4.8), we obtain

$$
\left|\tilde{y}_{2}^{u}-\tilde{y}_{1}^{u}\right|+\left|\tilde{y}_{2}^{c}-\tilde{y}_{2}^{c}\right| \leq O(\epsilon)\left(\left|y_{2}^{u}-y_{1}^{u}\right|+\left|y_{2}^{c}-y_{1}^{c}\right|\right) \text {. }
$$


Thus, from (4.7) and (4.9) we have

$$
\begin{aligned}
& \left|A\left(y_{2}^{u}+y_{2}^{c}\right)-A\left(y_{1}^{u}+y_{1}^{c}\right)\right| \\
& =\left|\left(\left.\Pi_{m_{2}}^{u} D T^{t_{1}}\left(m_{1}\right)\right|_{X_{m_{1}}^{u}}\right)^{-1}\left(\tilde{y}_{2}^{u}-\tilde{y}_{1}^{u}-\bar{y}_{2}^{u}+\bar{y}_{1}^{u}\right)+y_{2}^{u}-y_{1}^{u}\right| \\
& +\left|\left(\left.\Pi_{m_{2}}^{c} D T^{t_{1}}\left(m_{1}\right)\right|_{X_{m_{1}}^{c}}\right)^{-1}\left(\tilde{y}_{2}^{c}-\tilde{y}_{1}^{c}-\bar{y}_{2}^{u}+\bar{y}_{1}^{u}\right)+y_{2}^{c}-y_{1}^{c}\right| \\
& \leq O(\epsilon)\left(\left|y_{2}^{u}-y_{1}^{u}\right|+\left|y_{2}^{c}-y_{1}^{c}\right|\right) .
\end{aligned}
$$

Here Lemmas 3.4 and 3.5 are used to get a bound for $\left\|\left(\left.\Pi_{m_{2}}^{\alpha} D T^{t_{1}}\left(m_{1}\right)\right|_{X_{m_{1}}^{\alpha}}\right)^{-1}\right\|$ for $\alpha=u, c$. This shows that $A$ is a contraction by choosing $\epsilon^{*}$ sufficiently small. To complete the proof, we show that $A$ maps $\overline{X_{m_{1}}^{u}(\epsilon)} \oplus \overline{X_{m_{1}}^{c}(\epsilon)}$ into $X_{m_{1}}^{u}(\epsilon) \oplus X_{m_{1}}^{c}(\epsilon)$. We first estimate $A(0)$. Again using (4.5), we have

$$
\bar{y}^{\alpha}=\Pi_{m_{2}}^{\alpha}\left(T^{t_{1}}\left(x_{1}+y_{1}^{s}\right)-T^{t_{1}}\left(x_{1}\right)\right)
$$

for $\alpha=u, s, c$.

For $\alpha=u, c$ we estimate

$$
\begin{aligned}
\left|\bar{y}^{\alpha}\right| & =\left|\Pi_{m_{2}}^{\alpha}\left(T^{t_{1}}\left(x_{1}+y_{1}^{s}\right)-T^{t_{1}}\left(x_{1}\right)\right)\right| \\
& \leq O(\epsilon)\left|y_{1}^{s}\right| .
\end{aligned}
$$

We also estimate

$$
\begin{aligned}
\left|\bar{y}^{s}\right| & =\left|\Pi_{m_{2}}^{s}\left(T^{t_{1}}\left(x_{1}+y_{1}^{s}\right)-T^{t_{1}}\left(x_{1}\right)\right)\right| \\
& \leq\left\|\left.D T^{t_{1}}\left(m_{1}\right)\right|_{X_{m_{1}}^{s}}\right\|\left|y_{1}^{s}\right|+O(\epsilon)\left|y_{1}^{s}\right| .
\end{aligned}
$$

Hence, from (4.6) we obtain

$$
\left|\tilde{y}^{u}\right|+\left|\tilde{y}^{c}\right| \leq\left(\mu\left\|\left.D T^{t_{1}}\left(m_{1}\right)\right|_{X_{m_{1}}^{s}}\right\|+O(\epsilon)\right)\left|y_{1}^{s}\right| .
$$

Thus, for $\alpha=u, c$, we have

$$
\begin{aligned}
& \left|\left(\left.\Pi_{m_{2}}^{\alpha} D T^{t_{1}}\left(m_{1}\right)\right|_{X_{m_{1}}^{\alpha}}\right)^{-1}\left(\tilde{y}^{\alpha}-\bar{y}^{\alpha}\right)\right| \\
& \leq\left(\mu\left\|\left(\left.D T^{t_{1}}\left(m_{1}\right)\right|_{X_{m_{1}}^{\alpha}}\right)^{-1}\right\|\left\|\left.D T^{t_{1}}\left(m_{1}\right)\right|_{X_{m_{1}}}\right\|+O(\epsilon)\right)\left|y_{1}^{s}\right| \\
& \leq(\mu \lambda+O(\epsilon))\left|y_{1}^{s}\right| \\
& \leq(3 \mu \lambda+O(\epsilon)) \epsilon
\end{aligned}
$$

where Lemmas 3.4 and 3.5 are used.

Hence, for $\alpha=u, c$,

$$
\left|A^{\alpha}(0)\right| \leq(3 \mu \lambda+O(\epsilon)) \epsilon
$$

For any $y^{u}+y^{c} \in \overline{X_{m_{1}}^{u}(\epsilon)} \oplus \overline{X_{m_{1}}^{c}(\epsilon)}$, from (4.10) it follows that for $\alpha=u, c$,

$$
\begin{aligned}
\left|A^{\alpha}\left(y^{u}+y^{c}\right)\right| & \leq\left|A^{\alpha}\left(y^{u}+y^{c}\right)-A^{\alpha}(0)\right|+\left|A^{\alpha}(0)\right| \\
& \leq(3 \mu \lambda+O(\epsilon)) \epsilon<\epsilon
\end{aligned}
$$

provided that $\epsilon^{*}$ is sufficiently small since $0<\mu<\frac{1}{3}$ and $0<\lambda<1$. This proves the claim that $A$ is a contraction from $\overline{X_{m_{1}}^{u}(\epsilon)} \oplus \overline{X_{m_{1}}^{c}(\epsilon)}$ into $X_{m_{1}}^{u}(\epsilon) \oplus X_{m_{1}}^{s}(\epsilon)$. By the contraction mapping theorem, $A$ has a unique fixed point $y_{1}^{u}+y_{1}^{c} \in X_{m_{1}}^{u}(\epsilon) \oplus X_{m_{1}}^{c}(\epsilon)$, and thus, the corresponding $\bar{y}_{1}^{u}+\bar{y}_{1}^{c}+\bar{y}_{1}^{c}$ satisfies $\bar{y}_{1}^{u}+\bar{y}_{1}^{c}=f_{x_{2}}\left(\bar{y}_{1}^{s}\right)$; that is,

$$
T\left(x_{1}+y_{1}^{u}+y_{1}^{s}+y_{1}^{c}\right) \in \operatorname{graph}\left(f_{x_{2}}\right) .
$$

This completes the proof of the lemma. 
Define a map $\tilde{f}_{x_{1}}$ from $X_{m_{1}}^{s}(3 \epsilon)$ to $X_{m_{1}}^{u}(\epsilon) \oplus X_{m_{1}}^{c}(\epsilon)$ by

$$
\tilde{f}_{x_{1}}\left(y_{1}^{s}\right)=y_{1}^{u}+y_{1}^{c} \text {. }
$$

Lemma 4.3 shows that this map is well defined.

Clearly, by Lemma 3.3, we have

Lemma 4.4. $\tilde{f}_{x_{1}}(0)=0$ and Lip $\left(\tilde{f}_{x_{1}}\right) \leq \lambda_{1} \mu$, where $0<\lambda<\lambda_{1}<1$.

We now study the continuity of the fibers with respect to the base point $x$.

Let $x_{n}=m_{n}+x_{n}^{u}+x_{n}^{s} \in \Theta\left(X^{u}(\epsilon) \oplus X^{u}(\epsilon)\right)$, for $n=0,1,2, \ldots$, and suppose $x_{n} \rightarrow x_{0}$ as $n \rightarrow \infty$. Consider a sequence of stable fibers

$$
f_{x_{n}}: X_{m_{n}}^{s}(3 \epsilon) \rightarrow X_{m_{n}}^{u}(\epsilon) \oplus X_{m_{n}}^{c}(\epsilon)
$$

Definition. $f_{x_{n}}$ is said to be convergent to $f_{x_{0}}$ as $n \rightarrow \infty$ if for any $y_{n}^{s} \in X_{m_{n}}^{s}(3 \epsilon)$ satisfying

$$
x_{n}+y_{n}^{s} \rightarrow x_{0}+y_{0}^{s},
$$

then $x_{n}+y_{n}^{s}+f_{x_{n}}\left(y_{n}^{s}\right) \rightarrow x_{0}+y_{0}^{s}+f_{x_{0}}\left(y_{0}^{s}\right)$ as $n \rightarrow \infty$.

We denote this by

$$
f_{x_{n}} \rightarrow f_{x_{0}} \text { as } n \rightarrow \infty .
$$

Definition. $f_{x_{n}}$ is said to be uniformly convergent to $f_{x_{0}}$ if for any $\kappa>0$, there exists an integer $N>0$ such that for all $n>N$, if $y_{0}^{s} \in X_{m_{0}}^{s}(3 \epsilon)$ and $y_{n}^{s}=$ $\left(\left.\Pi_{m_{0}}^{s}\right|_{X_{m_{n}}^{s}}\right)^{-1} y_{0}^{s} \in X_{m_{n}}^{s}(3 \epsilon)$, then

$$
\left|x_{n}+y_{n}^{s}+f_{x_{n}}\left(y_{n}^{s}\right)-\left(x_{0}+y_{0}^{s}+f_{x_{0}}\left(y_{0}^{s}\right)\right)\right|<\kappa .
$$

Remark. The above definition of convergence is equivalent to saying that for any sequence $y_{n}^{s} \in X_{m_{n}}^{s}(3 \epsilon)$ satisfying $\left|\bar{y}_{n}^{s}-y_{0}^{s}\right| \rightarrow 0$, where $\bar{y}_{n}^{s}=\Pi_{m_{0}}^{s} y_{n}^{s}$, then

$$
\left(\Pi_{m_{0}}^{u} \oplus \Pi_{m_{0}}^{c}\right) f_{x_{n}}\left(y_{n}^{s}\right) \rightarrow f_{x_{0}}\left(y_{0}^{s}\right) \text {. }
$$

Let $x_{n}=m_{n}+x_{n}^{u}+x_{n}^{s} \in \Theta\left(X^{u}(\epsilon) \oplus X^{s}(\epsilon)\right)$ with $T^{t_{1}}\left(x_{n}\right) \in \Theta\left(X^{u}(\epsilon) \oplus X^{s}(\epsilon)\right)$ for $n=0,1,2, \ldots$, and $x_{n} \rightarrow x_{0}$. Denote $\tilde{x}_{n}=T^{t_{1}}\left(x_{n}\right)=\tilde{m}_{n}+\tilde{x}_{n}^{u}+\tilde{x}_{n}^{s}$. Consider a sequence of stable fibers $f_{\tilde{x}_{n}}$ with base point $\tilde{x}_{n}$. By Lemma 4.3 , for each $n$, there exists a unique stable fiber $\tilde{f}_{x_{n}}$ such that

$$
T^{t_{1}}\left(x_{n}+y_{n}^{s}+\tilde{f}_{x_{n}}\left(y_{n}^{s}\right)\right) \in \operatorname{graph}\left(f_{\tilde{x}_{n}}\right) .
$$

Note that $\tilde{x}_{n} \rightarrow \tilde{x}_{0}$ as $n \rightarrow \infty$. Then, we have

Lemma 4.5. (i) If $f_{\tilde{x}_{n}}$ converges to $f_{\tilde{x}_{0}}$, then $\tilde{f}_{x_{n}}$ converges to $\tilde{f}_{x_{0}}$;

(ii) If $f_{\tilde{x}_{n}}$ converges uniformly to $f_{\tilde{x}_{0}}$, then $\tilde{f}_{x_{n}}$ converges uniformly to $\tilde{f}_{x_{0}}$.

Proof. Let $y_{n}^{s} \in X_{m_{n}}^{s}(3 \epsilon)$. Suppose that $x_{n}+y_{n}^{s} \rightarrow x_{0}+y_{0}^{s}$ as $n \rightarrow \infty$. We shall show

$$
x_{n}+y_{n}^{s}+\tilde{f}_{x_{n}}\left(y_{n}^{s}\right) \rightarrow x_{0}+y_{0}^{s}+\tilde{f}_{x_{0}}\left(y_{0}^{s}\right) .
$$

For each $n$, by Lemma 4.3 , we write

$$
T^{t_{1}}\left(x_{n}+y_{n}^{s}+\tilde{f}_{x_{n}}\left(y_{n}^{s}\right)\right)=\tilde{x}_{n}+\tilde{y}_{n}^{u}+\tilde{y}_{n}^{s}+\tilde{y}_{n}^{c} .
$$

Note that $\tilde{y}_{n}^{u}+\tilde{y}_{n}^{c}=f_{\tilde{x}_{n}}\left(\tilde{y}_{n}^{s}\right)$.

Let $\tilde{f}_{x_{n}}\left(y_{n}^{s}\right)=y_{n}^{u}+y_{n}^{c}$. For $\tau \in[0,1]$, we denote

$$
\ell_{n}(\tau)=\tau\left(x_{n}+y_{n}^{u}+y_{n}^{s}+y_{n}^{c}\right)+(1-\tau)\left(x_{0}+y_{0}^{u}+y_{0}^{s}+y_{0}^{c}\right)
$$


A simple computation gives

$$
\left|\ell_{n}(\tau)-m_{0}\right| \leq C \epsilon,\left|T^{t_{1}}\left(m_{0}\right)-\tilde{m}_{0}\right| \leq C \epsilon,
$$

where $C$ is a positive constant. Thus, from (4.13), we obtain for $\alpha=u, s, c$,

$$
\begin{aligned}
\Pi_{\tilde{m}_{0}}^{\alpha}\left(\tilde{y}_{n}^{u}+\tilde{y}_{n}^{s}+\tilde{y}_{n}^{c}\right)-\tilde{y}_{0}^{\alpha} & \\
= & \left.\Pi_{\tilde{m}_{0}}^{\alpha}\left(\tilde{x}_{n}+\tilde{y}_{n}^{u}+\tilde{y}_{n}^{s}+\tilde{y}_{n}^{c}-\left(\tilde{x}_{0}+\tilde{y}_{0}^{u}+\tilde{y}_{0}^{s}+\tilde{y}_{0}^{c}\right)+\tilde{x}_{0}-\tilde{x}_{n}\right)\right) \\
= & \Pi_{\tilde{m}_{0}}^{\alpha}\left(\tilde{x}_{0}-\tilde{x}_{n}\right)+\Pi_{\tilde{m}_{0}}^{\alpha}\left(T^{t_{1}}\left(x_{n}+y_{n}^{s}+\tilde{f}_{x_{n}}\left(y_{n}^{s}\right)\right)\right. \\
& \left.\quad-T^{t_{1}}\left(x_{0}+y_{0}^{s}+\tilde{f}_{x_{0}}\left(y_{0}^{s}\right)\right)\right) \\
= & \Pi_{\tilde{m}_{0}}^{\alpha}\left(\tilde{x}_{0}-\tilde{x}_{n}\right) \\
& +\Pi_{\tilde{m}_{0}}^{\alpha} \int_{0}^{1} D T^{t_{1}}\left(\ell_{n}(\tau)\right)\left(x_{n}-x_{0}+y_{n}^{s}-y_{0}^{s}+\tilde{f}_{x_{n}}\left(y_{n}^{s}\right)-\tilde{f}_{x_{0}}\left(y_{0}^{s}\right)\right) d \tau \\
= & I_{n}+\left(\Pi_{\bar{m}_{0}}^{\alpha} D T^{t_{1}}\left(m_{0}\right)+O(\epsilon)\right) J_{n},
\end{aligned}
$$

where $\bar{m}_{0}=T^{t_{1}}\left(m_{0}\right), I_{n}$ is a quantity which approaches zero as $n \rightarrow \infty$, and $J_{n}=\Pi_{m_{0}}^{u} y_{n}^{u}-y_{0}^{u}+\Pi_{m_{0}}^{c} y_{n}^{c}-y_{0}^{c}$.

We want to show $J_{n} \rightarrow 0$ as $n \rightarrow \infty$.

For $\alpha \neq \alpha^{\prime}$, where $\alpha, \alpha^{\prime}=u, s, c$, we have

$$
\begin{aligned}
\left|\Pi_{\tilde{m}_{0}}^{\alpha} \tilde{y}_{n}^{\alpha^{\prime}}\right| & =\left|\left(\Pi_{\tilde{m}_{0}}^{\alpha}-\Pi_{\tilde{m}_{n}}^{\alpha}\right) \tilde{y}_{n}^{\alpha^{\prime}}\right| \\
& \leq 3 \epsilon|| \Pi_{\tilde{m}_{0}}^{\alpha}-\Pi_{\tilde{m}_{n}}^{\alpha} \| \rightarrow 0 \text { as } n \rightarrow \infty
\end{aligned}
$$

Hence, by (4.15) we obtain

$$
\Pi_{\tilde{m}_{0}}^{\alpha} \tilde{y}_{n}^{\alpha}-\tilde{y}_{0}^{\alpha}=\hat{I}_{n}+\left(\Pi_{\bar{m}_{0}}^{\alpha} D T^{t_{1}}\left(m_{0}\right)+O(\epsilon)\right) J_{n}
$$

where $\hat{I}_{n} \rightarrow 0$ as $n \rightarrow \infty$.

Let $\alpha=s$ in (4.16) and we estimate

$$
\left|\Pi_{\tilde{m}_{0}}^{s} \tilde{y}_{n}^{s}-\tilde{y}_{0}^{s}\right| \leq\left|\hat{I}_{n}\right|+O(\epsilon)\left|J_{n}\right| .
$$

Again, from (4.15) it follows that

$$
\begin{aligned}
&\left|\Pi_{\tilde{m}_{0}}^{u} \tilde{y}_{n}^{u}-\tilde{y}_{0}^{u}+\Pi_{\tilde{m}_{0}}^{c} \tilde{y}_{n}^{c}-\tilde{y}_{0}^{c}\right| \\
& \geq\left|\Pi_{\bar{m}_{0}}^{u} D T^{t_{1}}\left(m_{0}\right)\left(\Pi_{m_{0}}^{u} y_{n}^{u}-y_{0}^{u}\right)+\Pi_{\bar{m}_{0}}^{c} D T^{t_{1}}\left(m_{0}\right)\left(\Pi_{m_{0}} y_{n}^{c}-y_{0}^{c}\right)\right| \\
& \quad-\left|\hat{I}_{n}\right|-O(\epsilon)\left|J_{n}\right| \\
&=\left|D T^{t_{1}}\left(m_{0}\right)\left(\Pi_{m_{0}}^{u} y_{n}^{u}-y_{0}^{u}\right)+D T^{t_{1}}\left(m_{0}\right)\left(\Pi_{m_{0}}^{c} y_{n}^{c}-y_{0}^{c}\right)\right| \\
& \quad-\left|\hat{I}_{n}\right|-O(\epsilon)\left|J_{n}\right| \\
& \geq \frac{1}{C}\left|\Pi_{m_{0}}^{u} y_{n}^{u}-y_{0}^{u}+\Pi_{m_{0}}^{c} y_{n}^{c}-y_{0}^{c}\right|-\left|\hat{I}_{n}\right|-O(\epsilon)\left|J_{n}\right| \\
& \geq\left(\frac{1}{C}-O(\epsilon)\right)\left|J_{n}\right|-\left|\hat{I}_{n}\right|,
\end{aligned}
$$

where Lemma 3.4 and Lemma 3.5 are used.

Let $\bar{y}_{n}^{u}+\bar{y}_{n}^{c}=f_{\tilde{x}_{n}}\left(\left(\Pi_{\tilde{m}_{n}}^{s} \mid X_{\tilde{m}_{n}}^{s}\right)^{-1} \tilde{y}_{0}^{s}\right)$, which is well defined for large $n$. Since $f_{\tilde{x}_{n}} \rightarrow f_{\tilde{x}_{0}}$, we have

$$
\Pi_{\tilde{m}_{0}}^{u} \bar{y}_{n}^{u} \rightarrow \tilde{y}_{0}^{u} \text { and } \Pi_{\tilde{m}_{0}}^{c} \bar{y}_{n}^{c} \rightarrow \tilde{y}_{0}^{c}
$$

as $n \rightarrow \infty$. 
From (4.18), by using (4.17), we have for large $n$

$$
\begin{aligned}
& \left(C^{-1}-O(\epsilon)\right)\left|J_{n}\right|-\left|\hat{I}_{n}\right| \\
\leq & \left|\Pi_{\tilde{m}_{0}}^{u}\left(\tilde{y}_{n}^{u}-\bar{y}_{n}^{u}\right)+\Pi_{\tilde{m}_{0}}^{c}\left(\tilde{y}_{n}^{c}-\bar{y}_{n}^{c}\right)\right| \\
& +\left|\Pi_{\tilde{m}_{0}}^{u} \bar{y}_{n}^{u}-\tilde{y}_{0}^{u}\right|+\left|\Pi_{\tilde{m}_{0}}^{c} \bar{y}_{n}^{c}-\tilde{y}_{0}^{c}\right| \\
\leq & \mu\left(|| \Pi_{\tilde{m}_{0}}^{u}||+|| \Pi_{\tilde{m}_{0}}^{c} \|\right)\left|\tilde{y}_{n}^{s}-\left(\Pi_{\tilde{m}_{0}}^{s} \mid X_{\tilde{m}_{n}}^{s}\right)^{-1} \tilde{y}_{0}^{s}\right| \\
& +\left|\Pi_{\tilde{m}_{0}}^{u} \bar{y}_{n}^{u}-\tilde{y}_{0}^{u}\right|+\left|\Pi_{\tilde{m}_{0}}^{c} \bar{y}_{n}^{c}-\tilde{y}_{0}^{c}\right| \\
\leq & C\left(\left|\hat{I}_{n}\right|+O(\epsilon)\left|J_{n}\right|\right)+\left|\Pi_{\tilde{m}_{0}}^{u} \bar{y}_{n}^{u}-\tilde{y}_{0}\right|+\left|\Pi_{\tilde{m}_{0}}^{c} \bar{y}_{n}^{c}-\tilde{y}_{0}^{c}\right|,
\end{aligned}
$$

where $C$ is a positive constant depending only on projections.

Therefore, if $\epsilon^{*}$ is sufficiently small, $J_{n} \rightarrow 0$ as $n \rightarrow \infty$, which then implies $f_{x_{n}} \rightarrow f_{x_{0}}$. The uniform convergence follows from the same estimate (4.20). This completes the proof of the lemma.

This lemma is the key to showing the continuity of the stable foliation with respect to base point in $M$.

The next lemma is needed to establish the fact that the semiflow induces a graph transform which is a contraction on $\Gamma_{\epsilon}^{s s}$.

We first define a norm for a stable fiber as follows

$$
\left\|f_{x}\right\|=\sup _{\substack{y^{s} \in X_{m}^{s}(3 \epsilon) \\ y^{s} \neq 0}} \frac{\left|f_{x}\left(y^{s}\right)\right|}{\left|y^{s}\right|} .
$$

It is easy to see that $\Gamma_{\epsilon}^{s s}$ is complete under the norm

$$
\|F\|=\sup \left\{\left\|f_{x}\right\|: f_{x} \in F\right\} .
$$

For $x_{1} \in \Theta\left(X^{u}(\epsilon) \oplus X^{s}(\epsilon)\right)$ with $T^{t_{1}}\left(x_{1}\right) \in \Theta\left(X^{u}(\epsilon) \oplus X^{s}(\epsilon)\right)$, let $x_{2}=T^{t_{1}}\left(x_{1}\right)$. Given any two stable fibers at $x_{2}$,

$$
f_{x_{2}}, g_{x_{2}}
$$

by Lemma 4.3 , there exists two corresponding fibers $\tilde{f}_{x_{1}}$ and $\tilde{g}_{x_{1}}$ which satisfy (4.1). Furthermore, we have

Lemma 4.6. There exists $\epsilon^{*}>0$ such that if $0<\epsilon<\epsilon^{*}$, then

$$
\left\|\tilde{f}_{x_{1}}-\tilde{g}_{x_{1}}\right\| \leq \lambda_{1}\left\|f_{x_{2}}-g_{x_{2}}\right\|
$$

where $\lambda<\lambda_{1}<1$.

Proof. For any $y_{1}^{s} \in X_{m_{1}}^{s}(3 \epsilon)$, by Lemma 4.3 , there exist $y_{2}^{s}, \tilde{y}_{2}^{s}$ such that

$$
T^{t_{1}}\left(x_{1}+y_{1}^{s}+\tilde{f}_{x_{1}}\left(y_{1}^{s}\right)\right)=x_{2}+y_{2}^{s}+f_{x_{2}}\left(y_{2}^{s}\right),
$$

and

$$
T^{t_{1}}\left(x_{1}+y_{1}^{s}+\tilde{g}_{x_{1}}\left(y_{1}^{s}\right)\right)=x_{2}+\tilde{y}_{2}+g_{x_{2}}\left(\tilde{y}_{2}^{s}\right)
$$

Let

$$
\begin{aligned}
& y_{1}^{u}+y_{1}^{c}=\tilde{f}_{x_{1}}\left(y_{1}^{s}\right), \\
& y_{2}^{u}+y_{2}^{c}=f_{x_{2}}\left(y_{2}^{s}\right), \\
& \tilde{y}_{2}^{u}+\tilde{y}_{2}^{c}=g_{x_{2}}\left(\tilde{y}_{2}^{s}\right) .
\end{aligned}
$$


Then, from (4.21) and (4.22) it follows that

$$
\begin{aligned}
& \left(\tilde{y}_{2}^{u}+\tilde{y}_{2}^{s}+\tilde{y}_{2}^{c}\right)-\left(y_{2}^{u}+y_{2}^{s}+y_{2}^{c}\right) \\
& =D T^{t}\left(m_{1}\right)\left(\tilde{g}_{x_{1}}\left(y_{1}^{s}\right)-\tilde{f}_{x_{1}}\left(y_{1}^{s}\right)\right)+O(\epsilon)\left|\tilde{g}_{x_{1}}\left(y_{1}^{s}\right)-\tilde{f}_{x_{1}}\left(y_{1}^{s}\right)\right| .
\end{aligned}
$$

Applying the projection $\Pi_{m_{2}}^{\alpha}$ to (4.23), we obtain

$$
\begin{aligned}
\tilde{y}_{2}^{\alpha}-y_{2}^{\alpha}= & \Pi_{m_{2}}^{\alpha} D T^{t_{1}}\left(m_{1}\right)\left(\tilde{g}_{x_{1}}\left(y_{1}^{s}\right)-\tilde{f}_{x_{1}}\left(y_{1}^{s}\right)\right) \\
& +O(\epsilon)\left|\tilde{g}_{x_{1}}\left(y_{1}^{s}\right)-\tilde{f}_{x_{1}}\left(y_{1}^{s}\right)\right| .
\end{aligned}
$$

Let $\alpha=s$ in (4.24), then,

$$
\tilde{y}_{2}^{s}-y_{2}^{s}=O(\epsilon)\left|\tilde{g}_{x_{1}}\left(y_{1}^{s}\right)-\tilde{f}_{x_{1}}\left(y_{1}^{s}\right)\right| .
$$

On the other hand, from (4.24), by using (4.25), we obtain

$$
\begin{aligned}
& \left|g_{x_{2}}\left(y_{2}^{s}\right)-f_{x_{2}}\left(y_{2}^{s}\right)\right| \\
& \geq\left|g_{x_{2}}\left(\tilde{y}_{2}^{s}\right)-f_{x_{2}}\left(y_{2}^{s}\right)\right|-\left|g_{x_{2}}\left(\tilde{y}_{2}^{s}\right)-g_{x_{2}}\left(y_{2}^{s}\right)\right| \\
& \geq\left|\tilde{y}_{2}^{u}-y_{2}^{u}\right|+\left|\tilde{y}_{2}^{c}-y_{2}^{c}\right|-\mu\left|\tilde{y}_{2}^{s}-y_{2}^{s}\right| \\
& \geq\left|\Pi_{m_{2}}^{u} D T^{t_{1}}\left(m_{1}\right)\left(\tilde{y}_{1}^{u}-y_{1}^{u}\right)\right|+\left|\Pi_{m_{2}}^{c} D T^{t_{1}}\left(m_{1}\right)\left(\tilde{y}_{1}^{c}-y_{1}^{c}\right)\right| \\
& \quad-O(\epsilon)\left|\tilde{g}_{x_{1}}\left(y_{1}^{s}\right)-\tilde{f}_{x_{1}}\left(y_{1}^{s}\right)\right| .
\end{aligned}
$$

From (4.21) it follows that

$$
y_{2}^{s}=\Pi_{m_{2}}^{s} D T^{t_{1}}\left(m_{1}\right)\left(y_{1}^{s}+\tilde{f}_{x_{1}}\left(y_{1}^{s}\right)\right)+O(\epsilon)\left(\left|y_{1}^{s}\right|+\tilde{f}_{x_{1}}\left(y_{1}^{s}\right)\right)
$$

which implies

$$
\left|y_{2}^{s}\right| \leq\left\|\left.\Pi_{m_{2}}^{s} D T^{t_{1}}\left(m_{1}\right)\right|_{X_{m_{1}}^{s}}\right\|\left|y_{1}^{s}\right|+O(\epsilon)\left|y_{1}^{s}\right| .
$$

Therefore, by (4.26) together with Lemma 3.5, we obtain

$$
\frac{\left|\tilde{g}_{x_{1}}\left(y_{1}^{s}\right)-\tilde{f}_{x_{1}}\left(y_{1}^{s}\right)\right|}{\left|y_{1}^{s}\right|} \leq \lambda_{1} \frac{\left|g_{x_{2}}\left(y_{2}^{s}\right)-f_{x_{2}}\left(y_{2}^{s}\right)\right|}{\left|y_{2}^{s}\right|}
$$

provided that $\epsilon^{*}$ is sufficiently small. Thus,

$$
\left\|\tilde{g}_{x_{1}}-\tilde{f}_{x_{1}}\right\| \leq \lambda_{1}\left\|g_{x_{2}}-f_{x_{2}}\right\| \text {. }
$$

This completes the proof of the lemma.

Proof of Proposition 4.2. By Lemma 4.3, for $F \in \Gamma_{\epsilon}^{s s}$, we may define a family of stable fibers

$$
\tilde{F}=\left\{\tilde{f}_{x}: x \in \overline{W^{c u}(\epsilon)}\right\}
$$

which satisfies

$$
T^{t_{1}}\left(\operatorname{graph}\left(\tilde{f}_{x}\right)\right) \subset \operatorname{graph}\left(f_{T^{t_{1}}(x)}\right),
$$

and so induces a graph transform

$$
\mathcal{F}^{s s}(F)=\tilde{F} .
$$

Clearly, such $\tilde{F}$ is unique. To complete the proof, we must show that $\tilde{F} \in \Gamma_{\epsilon}^{s s}$. It is enough to show that $\tilde{f}_{x}$ is uniformly continuous in $x$. Since $f_{x}$ is uniformly continuous in $x$, by using Lemma 4.5 , we obtain that $\tilde{f}_{x}$ is uniformly continuous in $x$. This completes the proof. 
We now have the graph transform $\mathcal{F}^{s s}$ defined on $\Gamma_{\epsilon}^{s s}$. It follows from Lemma 4.6 that $\mathcal{F}^{s s}$ is a contraction, hence, it has a unique fixed point

$$
F^{s s}=\left\{f_{x}^{s s}: x \in W^{c s}(\epsilon)\right\}
$$

The next result shows that each fiber lies on the center-stable manifold $W^{c s}(\tilde{\epsilon})$.

Proposition 4.7. For each $x \in W^{c s}(\epsilon)$, graph $\left(f_{x}^{s s}\right) \subset W^{c s}(\tilde{\epsilon})$.

Proof. For any $x_{0} \in W^{c s}(\epsilon)$, let

$$
x_{k}=\left(T^{t_{1}}\right)^{k}\left(x_{0}\right) .
$$

Note that $x_{k} \in W^{c s}(\epsilon)$. We write

$$
x_{k}=m_{k}+x_{k}^{u}+x_{k}^{s}
$$

where $x_{k}^{\alpha} \in X_{m_{k}}^{\alpha}(\epsilon)$, for $\alpha=u, s$.

Let $y_{0, i}^{s} \in X_{m_{0}}^{s}(3 \epsilon)$, for $i=1,2$. From the invariance of $F^{s s}$, there exist $y_{k, i}^{s} \in$ $X_{m_{k}}^{s}(3 \epsilon)$ such that for $i=1,2$

$$
T^{k t_{1}}\left(x_{0}+y_{0, i}^{s}+f_{x_{0}}^{s s}\left(y_{0, i}^{s}\right)\right)=x_{k}+y_{k, i}^{s}+f_{x_{k}}^{s s}\left(y_{k, i}^{s}\right) .
$$

An elementary computation gives

$$
\left|y_{k, 2}^{s}-y_{k, 1}^{s}\right| \leq \lambda_{1}^{k}\left|y_{0,2}^{s}-y_{0,1}^{s}\right| \text { for all } k \geq 1
$$

provided that $\epsilon^{*}$ is sufficiently small. Therefore, Theorem A (2) completes the proof of the proposition.

The next lemma gives a geometric description of $F^{s s}$ in terms of cones. For $x_{0} \in W^{c s}(\epsilon)$, let $x_{k}=T^{k t_{1}}\left(x_{0}\right)$ for $k \geq 1$. Write $x_{k}$ as

$$
x_{k}=m_{k}+x_{k}^{u}+x_{k}^{s}
$$

where $m_{k} \in M, x_{k}^{\alpha} \in X_{m_{k}}^{\alpha}(\epsilon)$ for $\alpha=u, s$. Then,

Proposition 4.8. For $y_{0}^{\alpha} \in X_{m_{0}}^{\alpha}(3 \epsilon), \alpha=u, s, c, f_{x_{0}}^{s s}\left(y_{0}^{s}\right)=y_{0}^{u}+y_{0}^{c}$ if and only if

$$
T^{k t_{1}}\left(x_{0}+y_{0}^{u}+y_{0}^{s}+y_{0}^{c}\right)=x_{k}+y_{k}^{u}+y_{k}^{s}+y_{k}^{c}, k \geq 1,
$$

$\left|y_{k}^{\alpha}\right| \in X_{m_{k}}^{\alpha}(3 \epsilon)$, for $\alpha=u, s, c$, and

$$
\left|y_{k}^{u}\right|+\left|y_{k}^{c}\right| \leq \mu\left|y_{k}^{s}\right|
$$

Proof. Suppose that $f_{x_{0}}^{s s}\left(y_{0}^{s}\right)=y_{0}^{u}+y_{0}^{c}$. The invariance of $F^{s s}$ implies that

$$
y_{k}^{u}+y_{k}^{c}=f_{x_{k}}^{s s}\left(y_{k}^{s}\right)
$$

Hence, $\left|y_{k}^{u}\right|+\left|y_{k}^{c}\right| \leq \mu\left|y_{k}^{s}\right|$ and $\left|y_{k}^{\alpha}\right|<3 \epsilon$.

Now we prove the converse. For each $k>0$, since $\left|y_{k}^{u}\right|+\left|y_{k}^{c}\right| \leq \mu\left|y_{k}^{s}\right|$, we may choose a stable fiber $f_{x_{k}}$ such that

$$
y_{k}^{u}+y_{k}^{c}=f_{x_{k}}\left(y_{k}^{s}\right)
$$

Repeatedly using Lemma 4.3, we obtain a sequence of stable fibers, all depending upon $k$,

$$
f_{x_{k-1}}, \ldots, f_{x_{0}}
$$

such that for $1 \leq j \leq k$,

$$
T^{t_{1}}\left(\operatorname{graph}\left(f_{x_{j-1}}\right)\right) \subset T^{t_{1}}\left(\operatorname{graph}\left(f_{x_{j}}\right)\right),
$$


and $f_{x_{j}}\left(y_{j}^{s}\right)=y_{j}^{u}+y_{j}^{c}$. By using Lemma 4.6, we have

$$
\begin{aligned}
& \left|y_{0}^{u}+y_{0}^{c}-f_{x_{0}}^{s s}\left(y_{0}^{s}\right)\right| \\
& =\left|f_{x_{0}}\left(y_{0}^{s}\right)-f_{x_{0}}^{s s}\left(y_{0}^{s}\right)\right| \leq\left|y_{0}^{s}\right|\left\|f_{x_{0}}-f_{x_{0}}^{s s}\right\| \\
& \leq\left|y_{0}^{s}\right| \lambda_{1}\left\|f_{x_{1}}-f_{x_{1}}^{s s}\right\| \leq\left|y_{0}^{s}\right| \lambda_{1}^{k}\left\|f_{x_{k}}-f_{x_{k}}^{s s}\right\| \\
& \leq 2 \mu\left|y_{0}^{s}\right| \lambda_{1}^{k} \rightarrow 0 \text { as } k \rightarrow \infty
\end{aligned}
$$

which yields

$$
y_{0}^{u}+y_{0}^{c}=f_{x_{0}}^{s s}\left(y_{0}^{s}\right)
$$

This completes the proof.

For $x_{0}, \tilde{x}_{0} \in W^{c s}(\epsilon)$, write

$$
\begin{aligned}
& x_{0}=m_{0}+x_{0}^{u}+x_{0}^{s}=\tilde{x}_{0}+\tilde{y}_{0}^{u}+\tilde{y}_{0}^{s}+\tilde{y}_{0}^{c}, \\
& \tilde{x}_{0}=\tilde{m}_{0}+\tilde{x}_{0}^{u}+\tilde{x}_{0}^{s}=x_{0}+y_{0}^{u}+y_{0}^{s}+y_{0}^{c},
\end{aligned}
$$

where

$$
\begin{aligned}
& x_{0}^{\alpha} \in X_{m_{0}}^{\alpha}(\epsilon), \tilde{x}_{0}^{\alpha} \in X_{\tilde{m}_{0}}^{\alpha}(\epsilon) \text { for } \alpha=u, s, \\
& y_{0}^{\alpha} \in X_{m_{0}}^{\alpha} \text { and } \tilde{y}_{0}^{\alpha} \in X_{\tilde{m}_{0}}^{\alpha} \text { for } \alpha=u, s, c .
\end{aligned}
$$

Then,

Proposition 4.9. If $y_{0}^{\alpha} \in X_{m_{0}}^{\alpha}(3 \epsilon)$ and $\tilde{y}_{0}^{\alpha} \in X_{\tilde{m}_{0}}^{\alpha}(3 \epsilon)$ for $\alpha=u, s, c$, then $y_{0}^{u}+$ $y_{0}^{c}=f_{x_{0}}^{s s}\left(y_{0}^{s}\right)$ if and only if $\tilde{y}_{0}^{u}+\tilde{y}_{0}^{c}=f_{\tilde{x}_{0}}^{s s}\left(\tilde{y}_{0}^{s}\right)$.

Proof. It is enough to show that if $y_{0}^{u}+y_{0}^{c}=f_{x_{0}}^{s s}\left(y_{0}^{s}\right)$, then $\tilde{y}_{0}^{u}+\tilde{y}_{0}^{c}=f_{\tilde{x}_{0}}^{s s}\left(\tilde{y}_{0}^{s}\right)$.

For $k \geq 1$ let

$$
\begin{aligned}
& x_{k}=T^{k t_{1}}\left(x_{0}\right)=m_{k}+x_{k}^{u}+x_{k}^{s}, \\
& \tilde{x}_{k}=T^{k t_{1}}\left(\tilde{x}_{0}\right)=\tilde{m}_{k}+\tilde{x}_{k}^{u}+\tilde{x}_{k}^{s} .
\end{aligned}
$$

Note that $x_{k}^{\alpha} \in X_{m_{k}}^{\alpha}(\epsilon)$ and $\tilde{x}_{k}^{\alpha} \in X_{\tilde{m}_{k}}^{\alpha}(\epsilon)$ for $\alpha=u, s$. We write $\tilde{x}_{k}$ as

$$
\tilde{x}_{k}=x_{k}+y_{k}^{u}+y_{k}^{s}+y_{k}^{c}
$$

It follows from the invariance of $F^{s s}$ that

$$
y_{k}^{u}+y_{k}^{c}=f_{x_{k}}^{s s}\left(y_{k}^{s}\right)
$$

Let

$$
x_{k}=\tilde{x}_{k}+\tilde{y}_{k}^{u}+\tilde{y}_{k}^{s}+\tilde{y}_{k}^{c}
$$

Add (4.28) to (4.30) to obtain

$$
\tilde{y}_{k}^{u}+y_{k}^{u}+\tilde{y}_{k}^{s}+y_{k}^{s}+\tilde{y}_{k}^{c}+y_{k}^{c}=0
$$

Applying the projection $\Pi_{\tilde{m}_{k}}^{\alpha}$ to the above identity, we have for $\alpha=u, s, c$

$$
\tilde{y}_{k}^{\alpha}=-\Pi_{\tilde{m}_{k}}^{\alpha}\left(y_{k}^{u}+y_{k}^{s}+y_{k}^{c}\right) .
$$


Note that $\left|m_{k}-\tilde{m}_{k}\right| \leq 9 \epsilon$. Let $\alpha=s$ in (4.31). Then, using (4.27), (4.29), we obtain

$$
\begin{aligned}
\left|\tilde{y}_{k}^{s}\right| & \leq\left|y_{k}^{s}\right|+O(\epsilon)\left(\left|y_{k}^{u}\right|+\left|y_{k}^{s}\right|+\left|y_{k}^{c}\right|\right) \\
& \leq(1+O(\epsilon))\left|y_{k}^{s}\right| \\
& \leq \lambda_{1}^{k}(1+O(\epsilon))\left|y_{1}^{s}\right| \\
& <3 \epsilon
\end{aligned}
$$

provided that $\epsilon$ is sufficiently small.

Let $\alpha=u, c$ in (4.31). We have

$$
\begin{aligned}
\left|\tilde{y}_{k}^{\alpha}\right| & \leq\left|y_{k}^{\alpha}\right|+O(\epsilon)\left(\left|y_{k}^{u}\right|+\left|y_{k}^{s}\right|+\left|y_{k}^{c}\right|\right) \\
& \leq(\mu+O(\epsilon))\left|y_{k}^{s}\right| \\
& <\epsilon
\end{aligned}
$$

provided that $\epsilon$ is sufficiently small since $\mu<\frac{1}{3}$. Hence, $\left|\tilde{y}_{k}^{\alpha}\right|<3 \epsilon$ for $\alpha=u, s, c$.

To prove $\tilde{y}_{0}^{u}+\tilde{y}_{0}^{c}=f_{\tilde{x}_{0}}^{s s}\left(\tilde{y}_{0}^{s}\right)$, by Proposition 4.8 , it is sufficient to prove

$$
\left|\tilde{y}_{k}^{u}\right|+\left|\tilde{y}_{k}^{c}\right| \leq \mu\left|\tilde{y}_{k}^{s}\right| .
$$

From (4.31), we obtain for $k \geq 1$,

$$
\begin{aligned}
\left|\tilde{y}_{k}^{u}\right|+\left|\tilde{y}_{k}^{c}\right| & \leq\left|y_{k}^{u}\right|+\left|y_{k}^{c}\right|+O(\epsilon)\left|y_{k}^{s}\right| \\
& \leq\left(\lambda_{1} \mu+O(\epsilon)\right)\left|y_{k}^{s}\right|,
\end{aligned}
$$

where Lemma 4.4 is used. Again, from (4.31) we obtain

$$
\left|\tilde{y}_{k}^{s}\right| \geq(1-O(\epsilon))\left|y_{k}^{s}\right| \text {. }
$$

Therefore, (4.32) yields

$$
\left|\tilde{y}^{u}\right|+\left|\tilde{y}^{c}\right| \leq \frac{\lambda_{1} \mu+O(\epsilon)}{1-O(\epsilon)}\left|\tilde{y}_{k}^{s}\right| \leq \mu\left|\tilde{y}_{k}^{s}\right|
$$

provided that $\epsilon$ is sufficiently small. This completes the proof of the proposition.

Proposition 4.10. For $x_{0}=m_{0}+x_{0}^{u}+x_{0}^{s} \in W^{c s}(\epsilon)$, there exists a unique $y_{0}^{s} \in$ $X_{m_{0}}^{s}(3 \epsilon)$ such that

$$
x_{0}+y_{0}^{s}+f_{x_{0}}^{s s}\left(y_{0}^{s}\right)=m \in M
$$

and $x_{0} \in \operatorname{graph}\left(f_{m}^{s s}\right)$.

Proof. We first prove the uniqueness. Suppose there exist $y_{1}^{s}, y_{2}^{s} \in X_{m_{0}}^{s}(3 \epsilon)$ such that

$$
x_{0}+y_{i}^{s}+f_{x_{0}}^{s s}\left(y_{i}^{s}\right)=m_{i} \in M, \quad i=1,2 .
$$

Clearly, $\left|m_{0}-m_{i}\right| \leq 9 \epsilon$. Write $f_{x_{0}}^{s s}\left(y_{i}^{s}\right)=y_{i}^{u}+y_{i}^{c}$. By Lemma 3.2, we have

$$
\left|y_{1}^{c}-y_{2}^{c}-\left(m_{1}-m_{2}\right)\right| \leq O\left(\epsilon_{1}\right)\left|m_{1}-m_{2}\right|
$$

and since $f_{x_{0}}^{s s}$ is Lipschitz

$$
\begin{aligned}
\left|y_{1}^{s}-y_{2}^{s}\right| & \leq O\left(\epsilon_{1}\right)\left|m_{1}-m_{2}\right| \\
& \leq O\left(\epsilon_{1}\right)\left|y_{1}^{s}-y_{2}^{s}\right| .
\end{aligned}
$$

By choosing $\epsilon_{1}$ small enough, we have $y_{1}^{s}=y_{2}^{s}$, which implies $m_{1}=m_{2}$. 
Suppose that there exists $y_{0}^{s} \in X_{m_{0}}^{s}(3 \epsilon)$ such that $x_{0}+y_{0}^{s}+f_{x_{0}}^{s s}\left(y_{0}^{s}\right)=m \in M$. Write

$$
x_{0}=m+y^{s}+y^{u}+y^{c} .
$$

Applying Lemma 3.2 to $m$ and $x_{0}$, we obtain

$$
\left|y^{c}-\left(m_{0}-m\right)\right| \leq O\left(\epsilon_{1}\right)\left|m_{0}-m\right|,
$$

which gives

$$
\left|m_{0}-m\right| \leq\left(1+O\left(\epsilon_{1}\right)\right)\left|y^{c}\right|
$$

For $\alpha=u, s$, we have

$$
\begin{aligned}
\left|y^{\alpha}\right| & \leq O\left(\epsilon_{1}\right)\left|y^{c}\right|+\left\|\left(\Pi_{m_{0}}^{\alpha} \mid X_{m}^{u}\right)^{-1}\right\|\left|x_{0}^{\alpha}\right| \\
& \leq O\left(\epsilon_{1}\right) \epsilon+(1+O(\epsilon)) \epsilon<2 \epsilon,
\end{aligned}
$$

provided $\epsilon_{1}$ and $\epsilon$ are sufficiently small.

Note that $y^{s}+y^{u}+y^{c}=-\left(y_{0}^{s}+f_{x_{0}}^{s s}\left(y_{0}^{s}\right)\right)=-\left(y_{0}^{s}+y_{0}^{u}+y_{0}^{c}\right)$. Thus,

$$
\begin{aligned}
\left|y^{c}\right| & =\left|\Pi_{m}^{c}\left(y_{0}^{s}+y_{0}^{u}+y_{0}^{c}\right)\right| \\
& \leq O(\epsilon)\left(\left|y_{0}\right|^{s}+\left|y_{0}^{u}\right|+\left|y_{0}^{c}\right|\right)+\left|y_{0}^{c}\right| \\
& \leq O(\epsilon) \epsilon+\epsilon \leq 3 \epsilon,
\end{aligned}
$$

provided that $\epsilon$ is sufficiently small.

It follows from Proposition 4.9 that

$$
y^{u}+y^{c}=f_{m}^{s s}\left(y^{s}\right) .
$$

Finally, we show the existence of $y_{0}^{s}$.

For $y^{s} \in X_{m_{0}}^{s}(3 \epsilon)$. We write $f_{x_{0}}^{s s}\left(y^{s}\right)=y^{u}+y^{c}$ and

$$
x_{0}+y^{s}+f_{x_{0}}^{s s}\left(y^{s}\right)=m+\tilde{y}^{u}+\tilde{y}^{s},
$$

where $\tilde{y}^{\alpha} \in X_{m}^{\alpha}\left(\epsilon_{1}\right)$ for $\alpha=u, s$, by Lemma 3.6. Note that $\left|m-m_{0}\right| \leq r$. Here $\epsilon_{1}$ and $r$ are given in Section 3. Thus, from the choice of $r, \bar{y}^{\alpha}=\left(\left.\Pi_{m}^{\alpha}\right|_{X_{m_{0}}^{\alpha}}\right)^{-1} \tilde{y}^{\alpha}$ is well defined.

Define a map $\xi: \overline{X_{m_{0}}^{s}(3 \epsilon)} \rightarrow X_{m_{0}}^{s}$ by

$$
\xi\left(y^{s}\right)=y^{s}-\bar{y}^{s} .
$$

Applying Lemma 3.2 to $m+\tilde{y}^{s}+\tilde{y}^{u}$ and $m_{0}$, we obtain

$$
\left|y^{c}-\left(m-m_{0}\right)\right| \leq O\left(\epsilon_{1}\right)\left|m-m_{0}\right|,
$$

which implies

$$
\begin{aligned}
\left|m-m_{0}\right| & \leq\left(1+O\left(\epsilon_{1}\right)\right)\left|y^{c}\right| \\
& \leq \mu\left(1+O\left(\epsilon_{1}\right)\right)\left|y^{s}\right| \\
& \leq \epsilon,
\end{aligned}
$$

provided $\epsilon_{1}$ is sufficiently small. For $\alpha=u, s$, we have

$$
\left|\bar{y}^{\alpha}-\left(y^{\alpha}+x_{0}^{\alpha}\right)\right| \leq O\left(\epsilon_{1}\right)\left|y^{c}\right|,
$$

and hence,

$$
\left|\bar{y}^{\alpha}\right| \leq 5 \epsilon,
$$

provided that $\epsilon_{1}$ is small enough. In particular,

$$
\left|\bar{y}^{s}-y^{s}\right| \leq\left|x_{0}^{\alpha}\right|+O\left(\epsilon_{1}\right)\left|y^{c}\right|<3 \epsilon .
$$

So $\xi$ maps $\overline{X_{m_{0}}^{s}(3 \epsilon)}$ into $X_{m_{0}}^{s}(3 \epsilon)$. 
Next, we show that $\xi$ is a contraction. For $y_{i}^{s} \in \overline{X_{m_{0}}^{s}(3 \epsilon)}, i=1,2$, let

$$
x_{0}+y_{i}^{s}+f_{x_{0}}^{s s}\left(y_{i}^{s}\right)=m_{i}+\tilde{y}_{i}^{u}+\tilde{y}_{i}^{s}
$$

and

$$
\bar{y}_{i}^{\alpha}=\left(\left.\Pi_{m_{i}}\right|_{X_{m_{0}}^{\alpha}}\right)^{-1} \tilde{y}_{i}^{\alpha} \text { for } \alpha=u, s .
$$

By Lemma 3.2, we have

$$
\begin{aligned}
& \left|y_{1}^{s}-y_{2}^{s}-\left(\bar{y}_{1}^{s}-\bar{y}_{2}^{s}\right)\right|+\left|y_{1}^{u}-y_{2}^{u}-\left(\bar{y}_{1}^{u}-\bar{y}_{2}^{u}\right)\right| \\
& \leq O\left(\epsilon_{1}\right)\left|y_{1}^{c}-y_{2}^{c}\right|+O(\epsilon)\left(\left|\bar{y}_{1}^{s}-\bar{y}_{2}^{s}\right|+\left|\bar{y}_{1}^{u}-\bar{y}_{2}^{u}\right|\right),
\end{aligned}
$$

which gives

$$
\begin{aligned}
& \left|\bar{y}_{1}^{s}-\bar{y}_{2}^{s}\right|+\left|\bar{y}_{1}^{u}-\bar{y}_{2}^{u}\right| \\
& \quad \leq O\left(\epsilon_{1}\right)\left|y_{1}^{c}-y_{2}^{c}\right|+(1+O(\epsilon))\left(\left|y_{1}^{s}-y_{2}^{s}\right|+\left|y_{1}^{u}-y_{2}^{u}\right|\right) .
\end{aligned}
$$

Hence, from (4.33) and the Lipschitz condition, it follows that

$$
\begin{aligned}
\left|\xi\left(y_{1}^{s}\right)-\xi\left(y_{2}^{s}\right)\right| & =\left|y_{1}^{s}-y_{2}^{s}-\left(\bar{y}_{1}^{s}-\bar{y}_{2}^{s}\right)\right| \\
& \leq\left(O\left(\epsilon_{1}\right)+O(\epsilon)\right)\left|y_{1}^{s}-y_{2}^{s}\right| \\
& \leq \frac{1}{2}\left|y_{1}^{s}-y_{2}^{s}\right|,
\end{aligned}
$$

provided that $\epsilon_{1}$ and $\epsilon$ are sufficiently small.

Therefore, $\xi$ is a contraction map and has a unique fixed point $y_{0}^{s}$, which yields, $\bar{y}_{0}^{s}=0$ and hence, $\tilde{y}_{0}^{s}=0$. The fact that $m+\tilde{y}_{0}^{u} \in W^{c s}(\epsilon)$ locally a Lipschitz graph over $X_{m}^{c} \oplus X_{m}^{s}$, implies that $\tilde{y}_{0}^{u}=0$. Thus, $m=x_{0}+y_{0}^{s}+f_{x_{0}}^{s s}\left(y_{0}^{s}\right)$. The proof is complete.

Proposition 4.11. For each $x_{0} \in W^{c s}(\epsilon)$, there exists a unique $m \in M$ such that $x_{0} \in \operatorname{graph}\left(f_{m}^{s s}\right)$.

Proof. Proposition 4.10 gives the existence of $m$. Suppose that there exist $m_{1}, m_{2} \in$ $M$ such that

$$
x_{0} \in \operatorname{graph}\left(f_{m_{i}}^{s s}\right), \quad i=1,2 .
$$

Then $x_{0}=m_{i}+y_{i}^{s}+f_{m_{i}}^{s s}\left(y_{i}^{s}\right), i=1,2$, where $y_{i}^{s} \in X_{m_{i}}(3 \epsilon)$.

Let $y_{i}^{u}+y_{i}^{c}=f_{m_{i}}^{s s}\left(y_{i}^{s}\right)$. Note that $\left|m_{0}-m_{i}\right| \leq 6 \epsilon$. Applying Lemma 3.2 to $x_{0}$ and $m_{i}$, we obtain

$$
\left|y_{i}^{s}\right| \leq O\left(\epsilon_{1}\right) \epsilon+(1+O(\epsilon))\left|x_{0}^{\alpha}\right| \leq 2 \epsilon
$$

provided $\epsilon_{1}$ and $\epsilon$ are sufficiently small. Therefore, $\left|y_{i}^{\alpha}\right|<3 \epsilon$ for $\alpha=u, s$, and $c$.

Let

$$
m_{i}=x_{0}+\bar{y}_{i}^{u}+\bar{y}_{i}^{s}+\bar{y}_{i}^{c},
$$

where $\bar{y}_{i}^{\alpha} \in X_{m_{0}}^{\alpha}$. Then,

$$
\bar{y}_{i}^{u}+\bar{y}_{i}^{s}+\bar{y}_{i}^{c}+y_{i}^{u}+y_{i}^{s}+y_{i}^{c}=0 \text {. }
$$

Thus, for $\alpha=u, s, c$,

$$
\left|\bar{y}_{i}^{\alpha}\right|=O(\epsilon)\left(\left|y_{i}^{u}\right|+\left|y_{i}^{s}\right|+\left|y_{i}^{c}\right|\right)+\left|y_{i}^{\alpha}\right|<3 \epsilon
$$

provided $\epsilon$ is small enough.

By Proposition 4.9, $m_{1}, m_{2} \in \operatorname{graph}\left(f_{x_{0}}^{s s}\right)$, hence $m_{1}=m_{2}$ from Proposition 4.10. The proof is complete. 
From this proposition, we may define a projection from $W^{c s}(\epsilon)$ to $M$ by

$$
P^{s}\left(x_{0}\right)=m \text {. }
$$

Lemma 4.12. $P^{s}$ is continuous.

Proof. Suppose this is not true. Then there exist a constant $c$ and a sequence

$$
x_{n}=m_{n}+x_{n}^{s}+f_{m_{n}}^{s s}\left(x_{n}^{s}\right),
$$

where $x_{n}^{s} \in X_{m_{n}}^{s}(3 \epsilon)$, such that $x_{n} \rightarrow x_{0}$, but

$$
\left|m_{n}-m_{0}\right|>c>0
$$

where $m_{0}=P^{s}\left(x_{0}\right)$. Since $M$ is compact, we may assume $m_{n} \rightarrow \tilde{m}_{0} \in M$. Write

$$
x_{n}=\tilde{m}_{0}+\tilde{x}_{n}^{u}+\tilde{x}_{n}^{s}+\tilde{x}_{n}^{c} .
$$

Then $\tilde{x}_{n}^{\alpha} \rightarrow \tilde{x}_{0}^{\alpha} \in X_{\tilde{m}_{0}}^{\alpha}$ as $n \rightarrow \infty$. From the continuity of $f_{x}^{s s}$, we have $\tilde{x}_{0}^{u}+\tilde{x}_{0}^{c}=$ $f_{\tilde{m}_{0}}^{s s}\left(\tilde{x}_{0}^{s}\right)$, a contradiction to Proposition 4.10. The proof is complete.

Proposition 4.13. For all $m \in M$ and $t>0, T^{t}\left(\operatorname{graph}\left(f_{m}^{s s}\right)\right) \cap \Theta\left(X^{u}(\epsilon) \oplus X^{s}(\epsilon)\right) \subset$ $\operatorname{graph}\left(f_{T^{t} m}^{s s}\right)$.

Proof. For possibly much smaller $\epsilon$ and $x \in \operatorname{graph}\left(f_{m}^{s s}\right)$, we write

$$
x_{t}:=T^{t}(x)=\bar{m}_{t}+\bar{x}_{t}^{s}+\bar{x}_{t}^{u}+\bar{x}_{t}^{c}
$$

where $\bar{x}_{t}^{u}+\bar{x}_{t}^{c}=f_{\bar{m}_{t}}^{s s}\left(\bar{x}_{t}^{s}\right)$. The reason we may need smaller $\epsilon$ here is to guarantee that $x_{t}$ can be written in the above form.

Let $m_{t}=T^{t}(m)$. We also write

$$
x_{t}=m_{t}+x_{t}^{s}+x_{t}^{u}+x_{t}^{c},
$$

where $x_{t}^{\alpha} \in X_{m_{t}}^{\alpha}$ for $\alpha=u, s$, and $c$.

Note that, because of the invariance under $T^{t_{1}}$, the two representations coincide for integer multiples of $t_{1}$; that is,

$$
\bar{x}_{k t_{1}}^{\alpha}=x_{k t_{1}}^{\alpha}
$$

for all integers $k>0$ and $\alpha=u, s$, and $c$. Thus,

$$
\left|x_{k t_{1}}^{u}\right|+\left|x_{k t_{1}}^{c}\right| \leq \mu\left|x_{k t_{1}}^{s}\right| \text {. }
$$

It is easy to see that it is enough to show that

$$
m_{t}=\bar{m}_{t} \text { for } t \in\left(0, t_{1}\right) \text {. }
$$

We prove this by a contradiction. Suppose (4.35) does not hold. Then, there exists $t_{2} \in\left(0, t_{1}\right)$ such that

$$
m_{t_{2}} \neq \bar{m}_{t_{2}}
$$

Since $T^{t}$ is a diffeomorphism from $M$ onto $M, m_{k t_{1}+t_{2}} \neq \bar{m}_{k t_{1}+t_{2}}$ for all integers $k>0$.

By (4.27), we have

$$
\left|\bar{x}_{k t_{1}}^{s}\right| \leq 3 \lambda_{1}^{k} \epsilon
$$

For $k$ so large that $x_{k t_{1}}$ is sufficiently close to $m_{k t_{1}}$, we obtain

$$
\begin{aligned}
& \left|x_{k t_{1}+t_{2}}-m_{k t_{1}+t_{2}}\right| \\
& =\left|T^{t_{2}}\left(x_{k t_{1}}\right)-T^{t_{2}}\left(m_{k t_{1}}\right)\right| \\
& \leq \bar{C}\left|x_{k t_{1}}-m_{k t_{1}}\right| \leq \bar{C} \lambda_{1}^{k} \epsilon,
\end{aligned}
$$


where $\bar{C}$ depends on $t_{2}$. Here the fact that $m_{k t_{1}}=\bar{m}_{k t_{1}}$ is used.

Hence,

$$
\left|x_{k t_{1}+t_{2}}^{\alpha}\right| \leq \bar{C} \lambda_{1}^{k} \epsilon .
$$

Choose $k_{0}$ so large that $C \lambda_{1}^{k_{0}} \epsilon<\epsilon$. From Proposition 4.8 , there exists $k_{1}>k_{0}$ such that

$$
\left|x_{k t_{1}+t_{2}}^{u}\right|+\left|x_{k t_{1}+t_{2}}^{c}\right|>\mu\left|x_{k t_{1}+t_{2}}^{s}\right|
$$

for all integers $k \geq k_{1}$.

On the other hand, since $T^{2 t_{1}-t_{2}}$ satisfies (2.1) and (2.2), using Lemma 3.3 and (4.36), we obtain

$$
\left|x_{\left(k_{1}+2\right) t_{1}}^{u}\right|+\left|x_{\left(k_{1}+2\right) t_{1}}^{c}\right|>\mu\left|x_{\left(k_{1}+2\right) t_{1}}^{s}\right|,
$$

which contradicts (4.34). This completes the proof.

Proposition 4.14. (i) For $x, y \in W_{m}^{s s}(\epsilon)$, we have

$$
\left|T^{t}(x)-T^{t}(y)\right| \rightarrow 0
$$

exponentially as $t \rightarrow+\infty$. (ii) For $x \in W_{m}^{s s}(\epsilon), \bar{m} \in M$, and $\bar{m} \neq m$, we have

$$
\frac{\left|T^{t}(x)-T^{t}(m)\right|}{\left|T^{t}(x)-T^{t}(\bar{m})\right|} \rightarrow 0
$$

exponentially as $t \rightarrow+\infty$.

Proof. For $x=m+x^{u}+x^{s}+x^{c} \in W_{m}^{s s}(\epsilon)$, from the invariance of the stable foliation for $T^{t_{1}}$, we can write

$$
T^{k t_{1}}(x)=m_{k}+x_{k}^{u}+x_{k}^{s}+x_{k}^{c}
$$

where $m_{k}=T^{k t_{1}}(m), x_{k}^{\alpha} \in X_{m_{k}}^{\alpha}$ for $\alpha=u, s, c$, and $x_{k}^{u}+x_{k}^{c}=f_{m_{k}}^{s s}\left(x_{k}^{s}\right)$ with $\left|x_{k}^{s}\right|<3 \epsilon$. It follows from (4.27) that

$$
\left|x_{k}^{s}\right| \leq \lambda_{1}^{k}\left|x^{s}\right| \quad \text { for all } k \geq 0,
$$

which together with $\left|x_{k}^{u}\right|+\left|x_{k}^{c}\right| \leq \mu\left|x_{k}^{s}\right|$ gives

$$
\left|T^{k t_{1}}(x)-T^{k t_{1}}(m)\right|=\left|x_{k}^{u}+x_{k}^{c}+x_{k}^{s}\right| \leq(1+\mu) \lambda_{1}^{k}\left|x^{s}\right| .
$$

For each $t>t_{1}$, we write $t=k t_{1}+t_{2}$ for some $k>0$ and $t_{2} \in\left[0, t_{1}\right)$. By using (4.38) and Proposition 4.13, we have

$$
\begin{aligned}
& \left|T^{t}(x)-T^{t}(m)\right| \\
& =\left|T^{k t_{1}}\left(T^{t_{2}}(x)\right)-T^{k t_{1}}\left(T^{t_{2}}(m)\right)\right| \\
& \leq 3 \epsilon(1+\mu) \lambda_{1}^{k} \leq C e^{-\omega t},
\end{aligned}
$$

where $\omega=-\left(\ln \lambda_{1}\right) / t_{1}>0$, and $C=3 \epsilon(1+\mu) e^{\omega t_{2}}$. This completes the proof of (i).

Since $M$ is compact and $T^{t_{1}}$ is a diffeomorphism from $M$ onto $M$, for a given $\epsilon_{1}$ there are $0<r_{1}<r_{2}$ such that $B\left(m, r_{i}\right), i=1,2$, are $\eta$-neighborhoods satisfying

$$
\begin{aligned}
& M \cap B\left(T^{t_{1}}(m), r_{1}\right) \subset T^{t_{1}}\left(M \cap B\left(m, r_{2}\right)\right), \\
& T^{t_{1}}\left(M \cap B\left(m, r_{1}\right)\right) \subset M \cap B\left(T^{t_{1}}(m), r_{2}\right),
\end{aligned}
$$

and such that Lemma 3.2 can be applied to these $\eta$-neighborhoods. 
For $\bar{m} \in M$, denote $\bar{m}_{k}=T^{k t_{1}}(\bar{m})$ for $k=0,1, \cdots$. Suppose that $\bar{m}_{k+1} \in$ $B\left(m_{k+1}, r_{1}\right)$ which is an $\eta$-neighborhood of $m_{k}$. For $i=k-1, k$, we write

$$
\bar{m}_{i}=m_{i}+\bar{x}_{i}^{u}+\bar{x}_{i}^{s}+\bar{x}_{i}^{c} .
$$

Because of (4.39), we can apply Lemma 3.2 to obtain, for $i=k-1, k$,

$$
\left|\bar{m}_{i}-m_{i}\right| \leq C\left|\bar{x}_{i}^{c}\right|
$$

and

$$
\left|\bar{x}_{i}^{u}\right|+\left|\bar{x}_{i}^{s}\right| \leq C \epsilon_{1}\left|\bar{x}_{i}^{c}\right|
$$

Thus, by choosing $\epsilon_{1}$ and $\epsilon^{*}$ sufficiently small, we obtain

$$
\frac{\left|x_{k}^{s}\right|}{\left|\bar{x}_{k}^{c}\right|} \leq \lambda_{1} \frac{\left|x_{k-1}^{s}\right|}{\left|\bar{x}_{k-1}^{c}\right|} \text {. }
$$

By using (4.41), (4.42) and $\left|x_{i}^{u}+x_{i}^{c}\right| \leq \mu\left|x_{i}^{s}\right|$ and choosing $\epsilon_{1}$ small enough, we obtain for $i=k-1, k$

$$
\frac{1}{2} \frac{\left|x_{i}^{s}\right|}{\left|\bar{x}_{i}^{c}\right|} \leq \frac{\left|x_{i}^{u}+x_{i}^{s}+x_{i}^{c}\right|}{\left|\bar{x}_{i}^{u}+\bar{x}_{i}^{s}+\bar{x}_{i}^{c}\right|} \leq 2 \frac{\left|x_{i}^{s}\right|}{\left|\bar{x}_{i}^{c}\right|}
$$

For each $k>0$, we consider two cases:

Case 1: $\bar{m}_{i} \in B\left(m_{i}, r_{1}\right)$ for all $i=0,1, \cdots, k$.

Case 2: There is a $0 \leq k_{0} \leq k+1$ such that $\bar{m}_{i} \notin B\left(m_{i}, r_{1}\right)$ for $i=k_{0}+1, \cdots, k$, but $\bar{m}_{k_{0}} \in B\left(m_{k_{0}}, r_{1}\right)$.

For Case 1, it follows from (4.43) and (4.44) that

$$
\begin{aligned}
& \frac{\left|T^{k t_{1}}(x)-T^{k t_{1}}(m)\right|}{\left|T^{k t_{1}}(\bar{m})-T^{k t_{1}}(m)\right|} \\
& \leq 2 \frac{\left|x_{k}^{s}\right|}{\left|\bar{x}_{k}^{c}\right|} \leq 2 \lambda_{1}^{k} \frac{\left|x^{s}\right|}{\left|\bar{x}^{c}\right|} \leq \frac{12(1+\mu) \epsilon}{\bar{m}-m} \lambda_{1}^{k} .
\end{aligned}
$$

For Case 2, again from (4.43) and (4.44), by using (4.37), we obtain

$$
\begin{aligned}
& \frac{\left|T^{k t_{1}}(x)-T^{k t_{1}}(m)\right|}{\left|T^{k t_{1}}(\bar{m})-T^{k t_{1}}(m)\right|} \\
& \leq 2 \frac{\left|x_{k}^{s}\right|}{\left|\bar{x}_{k}^{c}\right|} \leq 2 \lambda_{1}^{k-k_{0}} \frac{\left|x_{k_{0}}^{s}\right|}{\left|\bar{x}_{k_{0}}^{c}\right|} \\
& \leq 4 \lambda_{1}^{k-k_{0}} \frac{\left|T^{\left(k_{0}\right) t_{1}}(x)-T^{\left(k_{0}\right) t_{1}}(m)\right|}{\left|T^{\left(k_{0}\right) t_{1}}(\bar{m})-T^{\left(k_{0}\right) t_{1}}(m)\right|} \\
& \leq \frac{12(1+\mu) \epsilon}{r_{1}} \lambda_{1}^{k} .
\end{aligned}
$$

Combining (4.45) and (4.46) gives for all $k>0$

$$
\frac{\left|T^{k t_{1}}(x)-T^{k t_{1}}(m)\right|}{\left|T^{k t_{1}}(\bar{m})-T^{k t_{1}}(m)\right|} \leq \frac{12(1+\mu) \epsilon}{\min \left\{r_{1}, \bar{m}-m\right\}} \lambda_{1}^{k},
$$

which together with the fact that $\inf \left\{\left|T^{t}(\bar{m})-T^{t}(m)\right|: 0 \leq t \leq t_{1}\right\}>0$ yields

$$
\frac{\left|T^{t}(x)-T^{t}(m)\right|}{\left|T^{t}(\bar{m})-T^{t}(m)\right|} \rightarrow 0, \quad \text { exponentially as } t \rightarrow \infty .
$$

Hence, using statement (i) in this proposition, we obtain (ii). This completes the proof. 
Combining all propositions together, we obtain Theorem 4.1.

\section{5. $C^{1}$ SMOothness of the STABle FOLIATION}

In this section, we prove the smoothness of $f_{m}^{s s}\left(y^{s}\right)$ in $y^{s}$. We take the approach used in BLZ1]. The basic idea is to find a candidate for the tangent bundle of the stable fiber, which is invariant under the linearization $D T^{t_{1}}$, then to prove it is tangent to the stable fiber. The arguments are based on the idea of Lipschitz jets, as in [HPS], but with a Lipschitz jet space which is different from that introduced in $\mathrm{HPS}$.

Let $Y$ and $Z$ be Banach spaces. For $y_{0} \in Y$ and $z_{0} \in Z$, consider two local maps

$$
g_{i}: U_{i} \rightarrow Z, \quad g_{i}\left(y_{0}\right)=z_{0}, \quad i=1,2,
$$

where $U_{i}$ is a neighborhood of $y_{0}$.

Define

$$
d\left(g_{1}, g_{2}\right)=\varlimsup_{y \rightarrow y_{0}} \frac{\left|g_{1}(y)-g_{2}(y)\right|}{\left|y-y_{0}\right|} .
$$

We shall see that $d\left(g_{1}, g_{2}\right)$ defines a metric on a certain quotient space.

If $d\left(g_{1}, g_{2}\right)=0$, we say that $g_{1}$ is equivalent to $g_{2}$. The equivalence class of all local maps equivalent to $g_{1}$ is called the Lipschitz jet of $g_{1}$ at $y_{0}$, which is denoted by $j_{1}=\left[g_{1}\right]$. We use $J\left(Y, Z ; y_{0}, z_{0}\right)$ to denote the set of all jets at $y_{0}$ carrying $y_{0}$ to $z_{0}$. For $j_{1}, j_{2} \in J\left(Y, Z ; y_{0}, z_{0}\right)$, we define

$$
d\left(j_{1}, j_{2}\right)=\varlimsup_{y \rightarrow y_{0}} \frac{\left|g_{1}(y)-g_{2}(y)\right|}{\left|y-y_{0}\right|}
$$

where $g_{1}$ and $g_{2}$ are representatives of $j_{1}$ and $j_{2}$, respectively. It is not hard to see that $d\left(j_{1}, j_{2}\right)$ does not depend on the choices of the representatives.

Consider the jet spaces

$$
\begin{aligned}
& J^{b}=\left\{j \in J\left(Y, Z ; y_{0}, z_{0}\right): d\left(j,\left[z_{0}\right]\right)<\infty\right\}, \\
& J^{c}=\left\{j \in J^{b}: j\right. \text { has a representative which is continuous } \\
& \text { in a neighborhood of } \left.y_{0}\right\}, \\
& J^{d}=\left\{j \in J^{b}: j \text { has a differentiable representative }\right\}, \\
& J^{a}=\left\{j \in J^{b}: j \text { has an affine representative }\right\} .
\end{aligned}
$$

Theorem on Lipschitz Jets. Let $y_{0}=0, z_{0}=0$. Then $J^{b}$ is a Banach space with norm $\|j\|=d(j, 0)$. The sets $J^{c}, J^{d}$ and $J^{a}$ are closed subspaces of $J^{b}$ and

$$
J^{b} \supset J^{c} \supset J^{d}=J^{a} .
$$

The above results are borrowed from [HPS].

For each $m \in M$, set

$$
\begin{aligned}
& J^{b}(m)=J^{b}\left(X_{m}^{s}, X_{m}^{c} ; 0,0\right), \\
& J^{c}(m)=J^{c}\left(X_{m}^{s}, X_{m}^{c} ; 0,0\right), \\
& J^{d}(m)=J^{d}\left(X_{m}^{s}, X_{m}^{c} ; 0,0\right), \\
& J^{a}(m)=J^{a}\left(X_{m}^{s}, X_{m}^{c} ; 0,0\right) .
\end{aligned}
$$


Let $\rho \in(1,2)$ be such that $\theta=\mu \rho<\frac{1}{2}$. For each fixed $m \in M$, we define a Lipschitz jet space

$$
J_{\theta}^{\ell}(m)=J_{\theta}^{\ell}\left(X_{m}^{s}, X_{m}^{c} ; 0,0\right)
$$

to be the space of jets which have a representative with Lipschitz constant $\theta$ in a neighborhood of 0 . Let $J_{\theta}^{\ell}=J_{\theta}^{\ell}\left(X^{s}, X^{c} ; 0,0\right)$ denote the Lipschitz jet bundle over $M$ with fiber $J_{\theta}^{\ell}(m)$.

Consider all maps from $W^{c s}(\epsilon)$ to $J_{\theta}^{\ell}$ which map points $x=m+x^{s}+f_{m}^{s s}\left(x^{s}\right)$ to jets $j$ in $J_{\theta}^{\ell}(m)$.

Define

$$
\Sigma_{\theta}^{s s, \ell}=\left\{\gamma: W^{c s}(\epsilon) \rightarrow J_{\theta}^{\ell} \text { with }\|\gamma\|<\infty\right\}
$$

where $\|\gamma\|=\sup \left\{\|\gamma(x)\|: x \in W^{c s}(\epsilon)\right\}$. Similarly, we may define $\Sigma_{\theta}^{s s, d}$ and $\Sigma_{\theta}^{s s, a}$.

For $x=m+x^{s}+f_{m}^{s s}\left(x^{s}\right) \in W^{c s}(\epsilon)$, from the invariance of $F^{s s}$, we may write

$$
T^{t_{1}}(x)=\tilde{x}=\tilde{m}+\tilde{x}^{s}+f_{\tilde{m}}^{s s}\left(\tilde{x}^{s}\right),
$$

where $\tilde{x}^{s} \in X_{\tilde{m}}^{s}(3 \epsilon)$ and $\tilde{m}=T^{t_{1}} m$.

We also write

$$
f_{m}^{s s}\left(x^{s}\right)=x^{u}+x^{c}
$$

and

$$
f_{\tilde{m}}^{s s}\left(\tilde{x}^{s}\right)=\tilde{x}^{u}+\tilde{x}^{c}
$$

Note that $x^{\alpha} \in X_{m}^{\alpha}(\epsilon)$ and $\tilde{x}^{\alpha} \in X_{\tilde{m}}^{\alpha}(\epsilon)$ for $\alpha=u, c$.

Let $\tilde{\gamma} \in \Sigma_{\theta}^{s s, \ell}$ be fixed and let $\tilde{g}: X_{\tilde{m}}^{s}(\tilde{r}) \rightarrow X_{\tilde{m}}^{c}$ be a Lipschitz representative of $\tilde{\gamma}(\tilde{x})$ such that for $y_{1}^{s}, y_{2}^{s} \in \tilde{X}_{\tilde{m}}^{s}(\tilde{r})$,

$$
\left|\tilde{g}\left(y_{1}^{s}\right)-\tilde{g}\left(y_{2}^{s}\right)\right| \leq \theta\left|y_{1}^{s}-y_{2}^{s}\right|
$$

where $\tilde{r}$ is a positive constant.

We shall construct $\gamma \in \Sigma_{\theta}^{s s, \ell}$ such that $\tilde{\gamma}$ is the image of $\gamma$ under $D T^{t_{1}}$ in a certain sense, which will be stated precisely, later. For a given $\tilde{g}$ satisfying the above properties, we want to find a Lipschitz map $g: X_{m}^{s}(r) \rightarrow X_{m}^{c}$ for some $r>0$ such that for each $y^{s} \in X_{m}^{s}(r)$, there exists $\tilde{y}^{s} \in X_{\tilde{m}}^{s}(\tilde{r})$ satisfying

$$
D T^{t_{1}}(x)\left(y^{u}+y^{s}+g\left(y^{s}\right)\right)=\tilde{y}^{u}+\tilde{y}^{s}+\tilde{g}\left(\tilde{y}^{s}\right)
$$

where $y^{u}=D f_{m}^{c s}\left(x^{s}, x^{c}\right)\left(y^{s}, y^{c}\right), y^{c}=g\left(y^{s}\right), \tilde{y}^{u}=D f_{\tilde{m}}^{c s}\left(x^{s}, x^{c}\right)\left(\tilde{y}^{s}, \tilde{y}^{c}\right), \tilde{y}^{c}=\tilde{g}\left(\tilde{y}^{s}\right)$, and $f_{m}^{c s}$ is the $C^{1}$ local representative of $W^{c s}(\epsilon)$ defined on $X_{m}^{c}\left(\rho^{-1} \epsilon_{1}\right) \oplus X_{m}^{s}\left(\rho^{-1} \epsilon_{1}\right)$ with Lipschitz constant $\theta$, and $\tilde{f}_{\tilde{m}}^{c s}$ is similar. To see this, for $y^{s} \in X_{m}^{s}(r)$, we define a map $E: \overline{X_{m}^{c}(r)} \rightarrow X_{m}^{c}$ for some small $r>0$, by

$$
\begin{aligned}
E\left(y^{c}\right)=\left(\left.\Pi_{\tilde{m}}^{c} D T^{t_{1}}(x)\right|_{X_{m}^{c}}\right)^{-1} & \left(\tilde{g}\left(\Pi_{\tilde{m}}^{s} D T^{t_{1}}(x)\left(y^{u}+y^{s}+y^{c}\right)\right)\right) \\
& \left.-\Pi_{\tilde{m}}^{c} D T^{t_{1}}(x)\left(y^{u}+y^{s}\right)\right)
\end{aligned}
$$

where $y^{u}=D f_{m}^{c s}\left(x^{s}, x^{c}\right)\left(y^{s}, y^{c}\right)$.

We shall see that $E$ is a contraction and its fixed point gives a solution of (5.3).

Lemma 5.2. There exists $\epsilon^{*}>0$ such that for $\epsilon<\epsilon^{*}$ if $x=m+x^{s}+f_{m}^{s s}\left(x^{s}\right) \epsilon$ $W^{c s}(\epsilon)$ and $\tilde{g}$ satisfies (5.2), then there exists $r^{*}=r^{*}(\tilde{r})>0$ such that for $r<r^{*}$, $E$ is a contraction on $\overline{X_{m}^{c}(r)}$. 
Proof. We first show that $E$ is well defined from $\overline{X_{m}^{c}(r)}$ into itself. Observe that for $y^{\alpha} \in \overline{X_{m}^{\alpha}(r)}, \alpha=u, s, c$,

$$
\left|\Pi_{\tilde{m}}^{s} D T^{t_{1}}(x)\left(y^{u}+y^{s}+y^{c}\right)\right| \leq C r \leq \tilde{r}
$$

provided that $r^{*}<\tilde{r} / C$. Thus, $\tilde{g}\left(\Pi_{\tilde{m}}^{s} D T^{t_{1}}(x)\left(y^{u}+y^{s}+y^{c}\right)\right)$ is well defined. From Lemma 3.5, $\left(\left.\Pi_{\tilde{m}}^{c} D T^{t_{1}}(x)\right|_{X_{m}^{c}}\right)^{-1}$ exists. Hence, $E\left(y^{c}\right)$ is well defined for $y^{c} \in \overline{X_{m}^{c}(r)}$.

Next, we show $\left|E\left(y^{c}\right)\right|<r$. Using (5.2) and Lemma 3.5, we obtain

$$
\begin{gathered}
\left|E\left(y^{c}\right)\right| \leq\left\|\left(\left.\Pi_{\tilde{m}}^{c} D T^{t_{1}}(x)\right|_{X_{m}^{c}}\right)^{-1}\right\|\left(\theta\left|\Pi_{\tilde{m}}^{s} D T^{t_{1}}(x)\left(y^{u}+y^{s}+y^{c}\right)\right|\right. \\
\left.+\left|\Pi_{\tilde{m}}^{c} D T^{t_{1}}(x)\left(y^{s}+y^{u}\right)\right|\right) \\
\leq\left\|\left(\left.\Pi_{\tilde{m}}^{c} D T^{t_{1}}(x)\right|_{X_{m}^{c}}\right)^{-1}\right\|\left(O(\epsilon)+\theta\left\|\left.\Pi_{\tilde{m}}^{s} D T^{t_{1}}(x)\right|_{X_{m}^{s}}\right\|\right) r \\
\leq(O(\epsilon)+\theta \lambda) r<r
\end{gathered}
$$

provided that $\epsilon^{*}$ is sufficiently small. Here $0<\lambda<1$ and $\theta<\frac{1}{2}$ are used.

Finally, we show that $E$ is a contraction. For $y_{1}^{c}, y_{2}^{c} \in \overline{X_{m}^{c}(r)}$, from (5.2), (5.4) and Lemma 3.5, we have

$$
\begin{aligned}
& \left|E\left(y_{1}^{c}\right)-E\left(y_{2}^{c}\right)\right| \\
& \leq\left\|\left(\left.\Pi_{\tilde{m}}^{c} D T^{t_{1}}(x)\right|_{X_{m}^{c}}\right)^{-1}\right\|\left(\theta\left|\Pi_{\tilde{m}}^{s} D T^{t_{1}}(x)\left(y_{1}^{u}-y_{2}^{u}+y_{1}^{c}-y_{2}^{c}\right)\right|\right. \\
& \left.\leq O(\epsilon)\left(\left|y_{1}^{u}-y_{2}^{u}\right|+\left|y_{1}^{c}-y_{2}^{c}\right|\right) \quad+\left|\Pi_{\tilde{m}}^{c} D T^{t_{1}}(x)\left(y_{1}^{u}-y_{2}^{u}\right)\right|\right) \\
& \leq O(\epsilon)\left|y_{1}^{c}-y_{2}^{c}\right| \leq \frac{1}{2}\left|y_{1}^{c}-y_{2}^{c}\right|,
\end{aligned}
$$

provided that $\epsilon^{*}$ is sufficiently small. This completes the proof.

By the contraction mapping theorem, we obtain that for each $y^{s} \in X_{m}^{s}(r), E$ has a unique fixed point $y^{c} \in \overline{X_{m}^{c}(r)}$, which defines a map from $X_{m}^{s}(r)$ to $\overline{X_{m}^{c}(r)}$. We denote it by $y^{c}=g\left(y^{s}\right)$. Clearly, $g$ satisfies (5.3). Furthermore, this function is a Lipschitz function with the Lipschitz constant $\theta$.

Lemma 5.3. There exists $\epsilon^{*}>0$ such that for $\epsilon<\epsilon^{*}$ if $x \in W^{c s}(\epsilon)$, then for $y_{1}^{s}, y_{2}^{s} \in X_{m}^{s}(r)$,

$$
\left|g\left(y_{1}^{s}\right)-g\left(y_{2}^{s}\right)\right| \leq \theta\left|y_{1}^{s}-y_{2}^{s}\right| .
$$

Proof. From the definition of $g$, using Lemma 3.5, it follows that

$$
\begin{aligned}
& \left|g\left(y_{1}^{s}\right)-g\left(y_{2}^{s}\right)\right| \\
& \leq\left\|\left(\left.\Pi_{\tilde{m}}^{c} D T^{t_{1}}(x)\right|_{X_{m}^{u}}\right)^{-1}\right\|\left(\theta\left|\Pi_{\tilde{m}}^{s} D T^{t_{1}}(x)\left(y_{1}^{u}-y_{2}^{u}+y_{1}^{s}-y_{2}^{s}+y_{1}^{c}-y_{2}^{c}\right)\right|\right. \\
& \left.+\left|\Pi_{\tilde{m}}^{c} D T^{t_{1}}(x)\left(y_{1}^{u}-y_{2}^{u}+y_{1}^{s}-y_{2}^{s}\right)\right|\right) \\
& \leq O(\epsilon)\left|y_{1}^{c}-y_{2}^{c}\right|+(O(\epsilon)+\theta \lambda)\left|y_{1}^{s}-y_{2}^{s}\right|,
\end{aligned}
$$

which implies that

$$
\left|g\left(y_{1}^{s}\right)-g\left(y_{2}^{s}\right)\right|=\left|y_{1}^{c}-y_{2}^{c}\right| \leq \frac{O(\epsilon)+\theta \lambda}{1-O(\epsilon)}\left|y_{1}^{s}-y_{2}^{s}\right| \leq \theta\left|y_{1}^{s}-y_{2}^{s}\right|
$$

by choosing $\epsilon^{*}$ small enough. The proof is complete. 
Next, we want to show that the jet equivalence class $[g]$ does not depend on the choice of $\tilde{g} \in \tilde{\gamma}(\tilde{x})$. Let $\tilde{h}$ be another Lipschitz representative of $\tilde{\gamma}(\tilde{x})$ satisfying

$$
\left|\tilde{h}\left(\tilde{y}_{1}^{s}\right)-\tilde{h}\left(\tilde{y}_{2}^{s}\right)\right| \leq \theta\left|\tilde{y}_{1}^{s}-\tilde{y}_{2}^{s}\right|
$$

for $\tilde{y}_{1}^{s}, \tilde{y}_{2}^{s} \in X_{\tilde{m}}^{s}\left(\tilde{r}_{0}\right)$, where $\tilde{r}_{0}$ is a positive constant. In the same way as above, define $h: X_{m}^{s}\left(r_{0}\right) \rightarrow X_{m}^{c}\left(r_{0}\right)$.

Lemma 5.4. $[g]=[h]$.

Proof. Let $y^{s} \in X_{m}^{s}(\hat{r})$, where $\hat{r}=\min \left\{r, r_{0}\right\}$. Set

$$
y_{1}^{c}=g\left(y^{s}\right), y_{2}^{c}=h\left(y^{s}\right)
$$

We want to show that

$$
\|[g]-[h]\|=\varlimsup_{y^{s} \rightarrow 0} \frac{\left|g\left(y^{s}\right)-h\left(y^{s}\right)\right|}{\left|y^{s}\right|}=0 .
$$

Note that from $[\tilde{g}]=[\tilde{h}],\left|\tilde{g}\left(\tilde{y}^{s}\right)-\tilde{h}\left(\tilde{y}^{s}\right)\right|=o\left(\left|\tilde{y}^{s}\right|\right)$ as $\tilde{y}^{s} \rightarrow 0$. From the definition of $g$ and $h$, by Lemma 3.5, we obtain

$$
\left|y_{1}^{c}-y_{2}^{c}\right| \leq O(\epsilon)\left(\left|y_{1}^{c}-y_{2}^{c}\right|\right)+o\left(\left|\Pi_{\tilde{m}}^{s} D T^{t_{1}}(x)\left(y_{1}^{u}+y^{s}+y_{1}^{c}\right)\right|\right),
$$

which, together with $\left|y_{1}^{c}\right| \leq \theta\left|y^{s}\right|$ and $\left|y_{1}^{u}\right| \leq \theta\left(\left|y^{s}\right|+\left|y_{1}^{c}\right|\right)$, implies that

$$
\left|y_{1}^{c}-y_{2}^{c}\right|=o\left(\left|y^{s}\right|\right)
$$

The proof is complete.

Thus, for each given $\tilde{\gamma} \in \Sigma_{\theta}^{s s, \ell}$ one may define $\gamma \in \Sigma_{\theta}^{s s, \ell}$ by $\gamma(x)=[g]$.

The following summarizes what we have so far:

Proposition 5.5. There exists $\epsilon^{*}$ such that if $\epsilon<\epsilon^{*}$, then for each $\tilde{\gamma} \in \Sigma_{\theta}^{s s, \ell}$, there exists a unique $\gamma \in \Sigma_{\theta}^{s s, \ell}$ such that DT ${ }^{t_{1}}$ maps $\gamma$ to $\tilde{\gamma}$ in the sense that (5.3) holds.

Define

$$
\mathcal{F}(\tilde{\gamma})=\gamma
$$

We claim

Lemma 5.6. $\mathcal{F}$ is a contraction on $\Sigma_{\theta}^{s s, \ell}$.

Proof. Let $\tilde{\gamma}_{1}, \tilde{\gamma}_{2} \in \Sigma_{\theta}^{s s, \ell}$. For $x=m+x^{s}+f_{m}^{s s}\left(x^{s}\right)$, we again write

$$
T^{t_{1}}(x)=\tilde{x}=\tilde{m}+\tilde{x}^{s}+f_{\tilde{m}}^{s s}\left(\tilde{x}^{s}\right) .
$$

By Proposition 5.5, there exist Lipschitz functions $g_{1}, g_{2}: X_{m}^{s}(r) \rightarrow X_{m}^{c}(r)$ such that for $i=1,2$,

$$
\left|g_{i}\left(y_{1}^{s}\right)-g_{i}\left(y_{2}^{s}\right)\right| \leq \theta\left|y_{1}^{s}-y_{2}^{s}\right|
$$

and

$$
\begin{aligned}
{\left[g_{1}\right] } & =\mathcal{F}\left(\tilde{\gamma}_{1}\right)(x), \\
{\left[g_{2}\right] } & =\mathcal{F}\left(\tilde{\gamma}_{2}\right)(x) .
\end{aligned}
$$


Let $y_{i}^{c}=g_{i}\left(y^{s}\right)$ and $y_{i}^{u}=D f_{m}^{c s}\left(x^{s}, x^{c}\right)\left(y^{s}, y_{i}^{c}\right)$ for $i=1,2$. From the definition of $g_{1}$ and $g_{2}$

$$
\begin{aligned}
& g_{1}\left(y^{s}\right)-g_{2}\left(y^{s}\right)=y_{1}^{c}-y_{2}^{c} \\
& =\left(\left.\Pi_{\tilde{m}}^{c} D T^{t_{1}}(x)\right|_{X_{m}^{c}}\right)^{-1}\left(\tilde{g}_{1}\left(\Pi_{\tilde{m}}^{s} D T^{t_{1}}(x)\left(y_{1}^{u}+y^{s}+y_{1}^{c}\right)\right)\right. \\
& \left.\quad-\tilde{g}_{2}\left(\Pi_{\tilde{m}}^{s} D T^{t_{1}}(x)\left(y_{2}^{u}+y^{s}+y_{2}^{c}\right)\right)-\Pi_{\tilde{m}}^{c} D T^{t_{1}}(x)\left(y_{1}^{u}-y_{2}^{u}\right)\right)
\end{aligned}
$$

where $\tilde{g}_{1}$ and $\tilde{g}_{2}$ are Lipschitz representatives of $\tilde{\gamma}_{1}(\tilde{x})$ and $\tilde{\gamma}_{2}(\tilde{x})$, respectively, at $\tilde{x}$ which satisfy (5.2). Using Lemma 3.5, we obtain

$$
\begin{aligned}
\left|y_{1}^{c}-y_{2}^{c}\right| \leq & \left\|\left(\left.\Pi_{\tilde{m}}^{c} D T^{t_{1}}(x)\right|_{X_{M}^{c}}\right)^{-1}\right\|\left(\theta\left|\Pi_{\tilde{m}}^{s} D T^{t_{1}}(x)\left(y_{1}^{u}-y_{2}^{u}+y_{1}^{c}-y_{2}^{c}\right)\right|\right. \\
& \left.+\left\|\left[\tilde{g}_{1}\right]-\left[\tilde{g}_{2}\right]\right\|\left|\Pi_{\tilde{m}}^{s} D T^{t_{1}}(x)\left(y_{2}^{u}+y^{s}+y_{2}^{c}\right)\right|\right) \\
& +o\left(\Pi_{\tilde{m}}^{s} D T^{t_{1}}(m)\left(y_{2}^{u}+y^{s}+y_{2}^{c}\right)\right)+O(\epsilon)\left|y_{1}^{c}-y_{2}^{c}\right| \\
\leq & O(\epsilon)\left|y_{1}^{c}-y_{2}^{c}\right|+(\lambda+O(\epsilon))\left\|\left[\tilde{g}_{1}\right]-\left[\tilde{g}_{2}\right]\right\|\left|y^{s}\right|+o\left(\left|y^{s}\right|\right),
\end{aligned}
$$

where $\left|y_{i}^{c}\right| \leq \theta\left|y^{s}\right|,\left|y_{i}^{u}\right| \leq \theta\left(\left|y^{s}\right|+\left|y_{i}^{c}\right|\right), i=1,2$ are used. Therefore,

$$
\left|y_{1}^{c}-y_{2}^{c}\right| \leq \frac{\lambda+O(\epsilon)}{1-O(\epsilon)}\left\|\left[\tilde{g}_{1}\right]-\left[\tilde{g}_{2}\right]\right\|\left|y^{s}\right|+o\left(\left|y^{s}\right|\right),
$$

which yields

$$
\left\|\left[g_{1}\right]-\left[g_{2}\right]\right\| \leq \frac{\lambda+O(\epsilon)}{1-O(\epsilon)}\left\|\left[\tilde{g}_{1}\right]-\left[\tilde{g}_{2}\right]\right\| .
$$

Since $0<\lambda<1$, for $\lambda<\lambda_{1}<1$, we may choose $\epsilon^{*}$ small enough so that for $\epsilon<\epsilon^{*}$

$$
\frac{\lambda+O(\epsilon)}{1-O(\epsilon)}<\lambda_{1} .
$$

Thus,

$$
\left\|\mathcal{F}\left(\tilde{\gamma}_{1}\right)-\mathcal{F}\left(\tilde{\gamma}_{2}\right)\right\| \leq \lambda_{1}\left\|\tilde{\gamma}_{1}-\tilde{\gamma}_{2}\right\|
$$

This completes the proof.

Our goal is to find a unique fixed point of $\mathcal{F}$ in $\Sigma_{\theta}^{s s, \ell}$. The difficulty here is that $\Sigma_{\theta}^{s s, \ell}$ may not be a complete space. On the other hand, from (5.7), we have

$$
\left\|\mathcal{F}^{k}\left(\tilde{\gamma}_{1}\right)-\mathcal{F}^{k}\left(\tilde{\gamma}_{2}\right)\right\| \leq \lambda_{1}^{k}\left\|\tilde{\gamma}_{1}-\tilde{\gamma}_{2}\right\|
$$

which yields that at each $x=m+x^{s}+f_{m}^{s s}\left(x^{s}\right) \in W^{c s}(\epsilon)$

$$
\mathcal{F}^{k}(\gamma)(x)
$$

is a Cauchy sequence in $J^{c}(m)$. Since $J^{c}(m)$ is a Banach space, we have

$$
\mathcal{F}^{k}(\gamma)(x) \rightarrow \gamma_{0}(x)
$$

where $\gamma_{0}(x) \in J^{c}\left(X_{m}^{s}, X_{m}^{c} ; 0,0\right)$. Clearly, the limit $\gamma_{0}$ is unique and does not depend on the initial choice of $\gamma$. We shall show that $\gamma_{0} \in \Sigma_{\theta}^{s s, \ell}$.

For each $x=m+x^{s}+f_{m}^{s s}\left(x^{s}\right) \in W^{c s}(\epsilon)$, let

$$
x^{u}+x^{c}=f_{m}^{s s}\left(x^{s}\right)
$$

Set

$$
f_{1}\left(y^{s}\right)=\Pi_{m}^{c}\left(f_{m}^{s s}\left(y^{s}+x^{s}\right)-f_{m}^{s s}\left(x^{s}\right)\right) .
$$


Then, we have $f_{1}(0)=0$ and

$$
\left|f_{1}\left(y_{1}^{s}\right)-f_{1}\left(y_{2}^{s}\right)\right| \leq \theta\left|y_{1}^{s}-y_{2}^{s}\right|
$$

for $y_{1}^{s}, y_{2}^{s} \in X_{m}^{s}(r)$ for some $r>0$. Thus, $f_{1}$ induces $\gamma_{1} \in \Sigma_{\theta}^{s s, l}$ by

$$
\gamma_{1}(x)=\left[f_{1}\right] \text {. }
$$

Lemma 5.7. $\mathcal{F}\left(\gamma_{1}\right)=\gamma_{1}$ and hence $\gamma_{0}=\gamma_{1}$.

Proof. For $x=m+x^{s}+f_{m}^{s s}\left(x^{s}\right) \in W^{c s}(\epsilon)$, we write

$$
T^{t_{1}}(x)=\tilde{x}=\tilde{m}+\tilde{x}^{s}+f_{\tilde{m}}^{s s}\left(\tilde{x}^{s}\right) .
$$

Let

$$
\tilde{f}\left(\tilde{y}_{s}\right)=\Pi_{\tilde{m}}^{c}\left(f_{\tilde{m}}^{s s}\left(\tilde{y}_{s}+\tilde{x}^{s}\right)-f_{\tilde{m}}^{s s}\left(\tilde{x}^{s}\right)\right) .
$$

Let $f$ be given by Lemma 5.2 through $\tilde{f}$. We want to show $\left[f_{1}\right]=[f]$. For $y^{s} \in$ $X_{m}^{s}(r)$ let

$$
\begin{aligned}
& y_{1}^{c}=f_{1}\left(y^{s}\right), \\
& y^{c}=f\left(y^{s}\right) .
\end{aligned}
$$

From the definition of $f$,

$$
\begin{array}{r}
y^{c}=\left(\left.\Pi_{\tilde{m}}^{c} D T^{t_{1}}(x)\right|_{X_{m}^{c}}\right)^{-1}\left(\tilde{f}\left(\Pi_{\tilde{m}}^{s} D T^{t_{1}}(x)\left(y_{1}^{u}+y^{s}+y^{c}\right)\right)\right. \\
\left.-\Pi_{\tilde{m}}^{c} D T^{t_{1}}(x)\left(y_{1}^{u}+y^{s}\right)\right),
\end{array}
$$

where $y_{1}^{u}=D f_{m}^{c s}\left(x^{s}, x^{c}\right)\left(y^{s}, y^{c}\right)$. On the other hand, from the invariance of $\left\{f_{m}^{s s}\right\}$, there exists $\tilde{y}^{s} \in X_{\tilde{m}}^{s}(\tilde{r})$ such that

$$
T^{t_{1}}\left(x+y^{u}+y^{s}+f_{1}\left(y^{s}\right)\right)=\tilde{x}+\tilde{y}^{u}+\tilde{y}^{s}+\tilde{f}\left(\tilde{y}^{s}\right)
$$

where

$$
\begin{aligned}
& y^{u}=\Pi_{m}^{u}\left(f_{m}^{s s}\left(y^{s}+x^{s}\right)-f_{m}^{s s}\left(x^{s}\right)\right)=f_{m}^{c s}\left(x^{s}+y^{s}, x^{c}+y^{c}\right)-f_{m}^{c s}\left(x^{s}, x^{c}\right), \\
& \tilde{y}^{u}=\Pi_{\tilde{m}}^{u}\left(f_{\tilde{m}}^{s s}\left(\tilde{y}^{s}+\tilde{x}^{s}\right)-f_{\tilde{m}}^{s s}\left(\bar{x}^{s}\right)\right) .
\end{aligned}
$$

By the Taylor expansion, we obtain

$$
\tilde{y}^{u}+\tilde{y}^{s}+\tilde{y}^{c}=D T^{t_{1}}(x)\left(y^{u}+y^{s}+y_{1}^{c}\right)+o\left(\left|y^{u}\right|+\left|y^{s}\right|+\left|y_{1}^{c}\right|\right),
$$

where $\tilde{y}^{c}=\tilde{f}\left(\tilde{y}^{s}\right)$.

Note that $\left|y^{u}\right|+\left|y_{1}^{c}\right| \leq \theta\left|y^{s}\right|$. Hence,

$$
\tilde{y}^{u}+\tilde{y}^{s}+\tilde{y}^{c}=D T^{t_{1}}(x)\left(y^{u}+y^{s}+y_{1}^{c}\right)+o\left(\left|y^{s}\right|\right)
$$

and for $\alpha=u, s, c$,

$$
\tilde{y}^{\alpha}=\Pi_{\tilde{m}}^{\alpha} D T^{t_{1}}(x)\left(y^{u}+y^{s}+y_{1}^{c}\right)+o\left(\left|y^{s}\right|\right) .
$$

In particular,

$$
\tilde{y}^{c}=\Pi_{\tilde{m}}^{c} D T^{t_{1}}(x)\left(y^{u}+y^{s}+y_{1}^{c}\right)+o\left(\left|y^{s}\right|\right),
$$

which yields

$$
\begin{aligned}
y_{1}^{c}= & \left(\left.\Pi_{\tilde{m}}^{c} D T^{t_{1}}(x)\right|_{X_{m}^{c}}\right)^{-1}\left(\tilde{y}^{c}-\Pi_{\tilde{m}}^{c} D T^{t_{1}}(x)\left(y^{u}+y^{s}\right)\right)+o\left(\left|y^{s}\right|\right) \\
= & \left(\left.\Pi_{\tilde{m}}^{c} D T^{t_{1}}(x)\right|_{X_{m}^{c}}\right)^{-1}\left(\tilde{f}\left(\tilde{y}^{s}\right)-\Pi_{\tilde{m}}^{c} D T^{t_{1}}(x)\left(y^{u}+y^{s}\right)\right)+o\left(\left|y^{s}\right|\right) \\
= & \left(\left.\Pi_{\tilde{m}}^{c} D T^{t_{1}}(x)\right|_{X_{m}^{c}}\right)^{-1}\left(\tilde{f}\left(\Pi_{\tilde{m}}^{s} D T^{t_{1}}(x)\left(y^{u}+y^{s}+y_{1}^{c}\right)\right)\right. \\
& \left.\quad-\Pi_{\tilde{m}}^{c} D T^{t_{1}}(x)\left(y^{u}+y^{s}\right)\right)+o\left(\left|y^{s}\right|\right) .
\end{aligned}
$$


Using (5.8) and Lemma 3.5, we obtain

$$
\left|y^{c}-y_{1}^{c}\right| \leq O(\epsilon)\left|y^{c}-y_{1}^{c}\right|+o\left(\left|y^{s}\right|\right) .
$$

Thus, by choosing $\epsilon^{*}$ small enough, we obtain

$$
\left|y^{c}-y_{1}^{c}\right| \leq o\left(\left|y^{s}\right|\right)
$$

that is,

$$
\left|f_{1}\left(y^{s}\right)-f\left(y^{s}\right)\right| \leq o\left(\left|y^{s}\right|\right)
$$

and hence,

$$
\left[f_{1}\right]=[f] .
$$

The uniqueness of the limit $\gamma_{0}$ implies $\gamma_{0}=\gamma_{1}$. This completes the proof.

We are now ready to show

Proposition 5.8. $f_{m}^{s s}\left(y^{s}\right)$ is $C^{1}$ in $y^{s}$ and $D f_{m}^{s s}\left(y^{s}\right)$ is uniformly continuous in $m$.

Proof. It is clear that from the definition of $\mathcal{F}$, particularly the definition of $E$, we have that if $\gamma \in \Sigma_{\theta}^{s s, a}$, then $\mathcal{F}(\gamma) \in \Sigma_{\theta}^{s s, a}$. Since $J^{a}(m)=J^{d}(m)$ is closed, $\gamma_{0}(x)=\gamma_{0}\left(m+x^{s}+f_{m}^{s s}\left(x^{s}\right)\right) \in J^{a}(m)$, where $\gamma_{0}$ is the limit of the Cauchy sequence $\mathcal{F}^{k}(\gamma)$. Since $\gamma_{0}=\gamma_{1}$ is unique, for $x=m+x^{s}+f_{m}^{s s}\left(x^{s}\right)$, we have $\left[f_{1}\right] \in J^{a}(m)$, that is, $\Pi_{m}^{c} f_{m}^{s s}\left(y^{s}\right)$ is differentiable at $x^{s}$, which together with the fact that $f_{m}^{c s}$ is $C^{1}$ yields that $f_{m}^{s s}$ is differentiable.

Finally, we want to show that $D f_{m}^{s s}\left(x^{s}\right)$ is continuous in $x^{s}$. It is enough to show that $D \Pi_{m}^{c} f_{m}^{s s}\left(x^{s}\right)$ is continuous.

Let $L\left(X^{s}, X^{c}\right)$ denote the vector bundle over $W^{c s}(\epsilon)$ with fiber $L\left(X_{m}^{s}, X_{m}^{c}\right)$ on $W^{c s}(\epsilon) \cap W_{m}^{s s}(3 \epsilon)$, where $L\left(X_{m}^{s}, X_{m}^{c}\right)$ is the Banach space of all bounded linear operators from $X_{m}^{s}$ to $X_{m}^{c}$.

Define the space

$$
\Lambda_{\theta}^{s s}=\left\{\ell: W^{c s}(\epsilon) \rightarrow L\left(X^{s}, X^{c}\right) \text { is a } C^{0} \text { section and }\|\ell\| \leq \theta\right\}
$$

with the norm

$$
\|\ell\|=\sup \left\{\|\ell(x)\|: x \in W^{c s}(\epsilon)\right\} .
$$

It is easy to check that $\Lambda_{\theta}^{s s}$ is a complete metric space. The space $\Lambda_{\theta}^{s s}$ may be regarded as a subset of $\Sigma_{\theta}^{s s, \theta}$ by identifying $\ell$ and $[\ell]$. As in [BLZ1], we have $\mathcal{F}: \Lambda_{\theta}^{s s} \rightarrow \Lambda_{\theta}^{s s}$ is a contraction and has a unique fixed point $\ell \in \Lambda_{\theta}^{s s}$. The uniqueness of $\gamma_{0}$ implies

$$
\gamma_{0}=[\ell],
$$

which yields that $D f_{1}$ is continuous. The proof of the uniform continuity of $D f_{m}^{s s}\left(y^{s}\right)$ in $m$ follows in the same fashion as in Lemma 4.5. The proof is complete.

For $m \in M$, define

$$
W_{m}^{s s}(\epsilon)=\left\{m+x^{s}+f_{m}^{s s}\left(x^{s}\right) \in W^{c s}(\epsilon):\left|x^{s}\right|<3 \epsilon\right\},
$$

i.e., $W_{m}^{s s}(\epsilon)$ is the intersection of the center-stable manifold of size $\epsilon$ and the stable fiber at $m$.

The next result gives a geometric structure of the stable fibers. 
Proposition 5.9. Let $Q_{m_{0}}=\left\{x^{s} \in X_{m_{0}}^{s}(3 \epsilon): m_{0}+x^{s}+f_{m_{0}}^{s s}\left(x^{s}\right) \in W^{c s}(\epsilon)\right\}$. Then $Q_{m_{0}}$ is star-shaped.

Proof. For any $x^{s} \in X_{m_{0}}^{s}$ with $\left|x^{s}\right|=1$ and $\tau \in[0,3 \epsilon]$, we write

$$
m_{0}+\tau x^{s}+f_{m_{0}}^{s s}\left(\tau x^{s}\right)=m_{\tau}+\Pi_{m_{\tau}}^{u} x_{\tau}^{u}+\Pi_{m_{\tau}}^{s} x_{\tau}^{s},
$$

where $x_{\tau}^{\alpha} \in X_{m_{0}}^{\alpha}$ for $\alpha=u, s$. It is enough to show that $\left|\Pi_{m_{\tau}}^{s} x_{\tau}^{s}\right|$ is increasing in $\tau$. Computing the derivative of (5.9) with respect to $\tau$, we have

$$
\begin{aligned}
x^{s}+D f_{m_{0}}^{s s}\left(\tau x^{s}\right) x^{s}= & \bar{y}^{c}+D \Pi_{m_{\tau}}^{u}\left(\bar{y}^{c}\right) x_{\tau}^{u} \\
& +D \Pi_{m_{\tau}}^{s}\left(\bar{y}^{c}\right) x_{\tau}^{s}+\Pi_{m_{\tau}}^{u} y^{u}+\Pi_{m_{\tau}}^{s} y^{s},
\end{aligned}
$$

where $\bar{y}^{c}=\frac{d m_{\tau}}{d \tau} \in X_{m_{\tau}}^{c}$ and $y^{\alpha}=\frac{d x_{\tau}^{\alpha}}{d \tau} \in X_{m_{0}}^{\alpha}$ for $\alpha=u, s$. Note that

$$
\left\|D f_{m_{0}}^{s s}\left(\tau x^{s}\right)\right\|<\mu \text {. }
$$

Applying $\Pi_{m_{0}}^{s}, \Pi_{m_{0}}^{u}$, and $\Pi_{m_{0}}^{c}$ to (5.10), respectively, we have

$$
\begin{gathered}
y^{s}=x^{s}+O(\epsilon)\left(\bar{y}^{c}+y^{u}+y^{s}\right), \\
\left|y^{u}\right|<\mu\left|x^{s}\right|+O(\epsilon)\left(\left|\bar{y}^{c}\right|+\left|y^{s}\right|\right),
\end{gathered}
$$

and

$$
\left|\bar{y}^{c}\right|<\mu\left|x^{s}\right|+O(\epsilon)\left(\left|y^{u}\right|+\left|y^{s}\right|\right) .
$$

Therefore,

$$
y^{s}=x^{s}+O(\epsilon) x^{s} .
$$

Thus,

$$
\begin{aligned}
\left(\Pi_{m_{\tau}}^{s} x_{\tau}^{s}\right)^{\prime} & =D \Pi_{m_{\tau}}^{s}\left(\bar{y}^{c}\right) x_{\tau}^{s}+\Pi_{m_{\tau}}^{s} y^{s} \\
& =x^{s}+O(\epsilon) x^{s} \\
& =\Pi_{m_{\tau}}^{s} x_{\tau}^{s}+O(\epsilon) \Pi_{m_{\tau}}^{s} x_{\tau}^{s},
\end{aligned}
$$

which yields, by using the Taylor expansion, that $\left|\Pi_{m_{\tau}}^{s} x_{\tau}^{s}\right|$ is increasing in $\tau$. The proof is complete.

Summarizing the results we have obtained so far, gives Theorem 2.2.

\section{6. $C^{1}$ Unstable Foliation}

Generally, a few modifications are needed to extend our results in Sections 4 and 5 to the case of $W^{c u}(\epsilon)$. Here we present an outline of these modifications. The most significant differences are the definition of the graph transform and the associated spaces. We shall leave the details to the interested reader.

Corresponding to the idea of stable fiber, we define the following:

Definition. Let $x=m+x^{u}+x^{s} \in \Theta\left(X^{u}(\epsilon) \oplus X^{s}(\epsilon)\right)$. A map $f_{x}: X_{m}^{u}(3 \epsilon) \rightarrow$ $X_{m}^{s}(\epsilon) \oplus X_{m}^{c}(\epsilon)$ is called an unstable fiber if

(1) $f_{x}(0)=0$

(2) $f_{x}$ is Lipschitz continuous with Lipschitz constant $\operatorname{Lip}\left(f_{x}\right)<\mu$. 
We shall construct an unstable invariant foliation in the following space

$$
\begin{aligned}
\Gamma_{\epsilon}^{u u}=\left\{F=\left\{f_{x}\right\}_{x \in \overline{W^{c u}}(\epsilon)}\right. & : f_{x} \text { is an unstable fiber and } \\
& \text { is uniformly continuous in } x\} .
\end{aligned}
$$

In order to establish Theorem 2.3, we first construct an invariant foliation with Lipschitz fibers, then show the fibers are $C^{1}$ and uniformly continuous with respect to base points. The outline of the approach is as follows: The first step is to construct $\tilde{F} \in \Gamma_{\epsilon}^{u u}$ for each $F \in \Gamma_{\epsilon}^{u u}$ such that $\tilde{F}$ is the image of $F$ under the time- $t_{1}$ map $T^{t_{1}}$ in a certain sense. This defines a graph transform

$$
\mathcal{F}(F)=\tilde{F} .
$$

In the case of the stable foliation, we constructed a preimage $\tilde{F}$ of $F$ instead of the image under $T^{t_{1}}$.

The next step is to show that $\mathcal{F}$ is a contraction in $\Gamma_{\epsilon}^{u u}$ under the norm

$$
\|F\|=\sup _{x \in \overline{W^{c u}}(x)}\left\|f_{x}\right\|
$$

where

$$
\left\|f_{x}\right\|=\sup _{y^{u} \in X^{u}(3 \epsilon), y_{u} \neq 0} \frac{\left|f_{x}\left(y^{u}\right)\right|}{\left|y^{u}\right|} .
$$

Thus, $\mathcal{F}$ has a unique fixed point $F^{u u}=\left\{f_{x}^{u u}\right\} \in \Gamma_{\epsilon}^{u u}$.

The third step is to establish properties analogous to those for the stable foliation.

The final step is to show that each unstable fiber $f_{m}^{u u}$ is $C^{1}$ and continuous in $m$.

Let us first look at how to construct $\mathcal{F}$. Let $\tilde{x} \in \Theta\left(X^{u}(\epsilon) \oplus X^{s}(\epsilon)\right)$ be such that there exists $x \in \Theta\left(X^{u}(\epsilon) \oplus X^{s}(\epsilon)\right)$ satisfying $\tilde{x}=T^{t_{1}}(x)$. For a given unstable fiber

$$
f_{x}: X_{m}^{u}(3 \epsilon) \rightarrow X^{s}(\epsilon) \oplus X^{c}(\epsilon),
$$

we first want to construct an unstable fiber $\tilde{f}_{\tilde{x}}$ at $\tilde{x}$ such that

$$
T^{t_{1}}\left(\operatorname{graph}\left(f_{x}\right)\right) \supset \operatorname{graph}\left(\tilde{f}_{\tilde{x}}\right) .
$$

To do this, for each $\tilde{y}^{u} \in X_{\tilde{m}}^{u}(3 \epsilon)$, we define a map $A$ from $X_{m}^{u}(3 \epsilon)$ to $X_{m}^{u}$ by

$$
A\left(y^{u}\right)=\left(\left.\Pi_{\tilde{m}}^{u} D T^{t_{1}}\left(m_{1}\right)\right|_{X_{m}^{u}}\right)^{-1}\left(\tilde{y}^{u}-\Pi_{\tilde{m}}^{u}\left(T^{t_{1}}\left(x+y^{u}+f_{x}\left(y^{u}\right)\right)-\tilde{x}\right)\right)+y^{u} .
$$

In the same fashion as for $A$ in Lemma 4.3, one may show that there exists $\epsilon^{*}>0$ such that if $\epsilon<\epsilon^{*}$, then $A$ is a contraction from $\overline{X_{m}^{u}(3 \epsilon)}$ into $X_{m}^{u}(3 \epsilon)$, hence, has a unique fixed point $y^{u} \in X_{m}^{u}(3 \epsilon)$. For $\alpha=u, s, c$ let

$$
\bar{y}^{\alpha}=\Pi_{\tilde{m}}^{\alpha}\left(T^{t_{1}}\left(x+y^{u}+f_{x}\left(y^{u}\right)\right)-\tilde{x}\right) .
$$

Note that $\tilde{y}^{u}=\bar{y}^{u}$. Define

$$
\tilde{f}_{\tilde{x}}\left(\bar{y}^{u}\right)=\bar{y}^{s}+\bar{y}^{c} .
$$

Then, $\tilde{f}_{\tilde{x}}$ is an unstable fiber with Lipschitz constant $\lambda_{1} \mu$. Let $F \in \Gamma_{\epsilon}^{u u}$. For $\tilde{x} \in W^{c u}(\epsilon)$, by the negative invariance property of $W^{c u}(\epsilon)$, there exists a unique $x \in W^{c u}(\epsilon)$ such that $T^{t_{1}}(x)=\tilde{x}$. The above discussion produces a unique unstable fiber $\tilde{f}_{\tilde{x}}$ at $\tilde{x}$ such that

$$
T^{t_{1}}\left(\operatorname{graph}\left(f_{x}\right)\right) \supset \operatorname{graph}\left(\tilde{f}_{\tilde{x}}\right) .
$$


Let $\tilde{F}=\left\{\tilde{f}_{\tilde{x}}: \tilde{x} \in W^{c u}(\epsilon)\right\}$. Thus, one may define a graph transform $\mathcal{F}$ from $\Gamma_{\epsilon}^{u u}$ into itself by

$$
\mathcal{F}(F)=\tilde{F} .
$$

Using a similar argument to that used in Lemma 4.6, one can show that $\mathcal{F}$ is a contraction, hence has a unique fixed point $F^{u u} \in \Gamma_{\epsilon}^{u u}$. Propositions 4.7-4.11 carry over with a little modification in the case of the unstable foliation. To show $f_{m}^{u u}$ is $C^{1}$, we define

$$
\Sigma_{\theta}^{u u, l}=\left\{\gamma: W^{c u}(\epsilon) \rightarrow J_{\theta}^{l}\left(X^{u}, X^{c} ; 0,0\right) \text { with }\|\gamma\|<\infty\right\} .
$$

In order to show the smoothness of $f_{m}^{u u}$, we need to find a candidate for the tangent bundle of $f_{m}^{u u}$, then to show it indeed is the tangent bundle. The approach is the same as that for the smoothness of the center unstable manifold in [BLZ1], which is modified from the stable case. The significant difference is the graph transform. For each $\gamma \in \Sigma_{\theta}^{u u, \ell}$, we want to construct $\tilde{\gamma} \in \Sigma_{\theta}^{u u, \ell}$ such that $\tilde{\gamma}$ is the image of $\gamma$ under $D T^{t_{1}}$ in a certain sense, defining a graph transform

$$
\mathcal{F}(\gamma)=\tilde{\gamma}
$$

For each $\tilde{x}=\tilde{m}+\tilde{x}^{u}+\tilde{x}^{s}+\tilde{x}^{c} \in W^{c u}(\epsilon)$, where $\tilde{x}^{s}+\tilde{x}^{c}=f_{\tilde{m}}^{u u}\left(\tilde{x}^{u}\right)$, by Theorem $A$, there exists a unique point $x=m+x^{u}+x^{s}+x^{c} \in W^{c u}(\epsilon)$, where $x^{s}+x^{c}=f_{\tilde{m}}^{u u}\left(x^{u}\right)$, such that $\tilde{x}=T^{t_{1}}(x)$. For $\gamma \in \Sigma_{\theta}^{u u, \ell}$, let $g$ be a Lipschitz representative of $\gamma(x)$. We want to construct a Lipschitz map

$$
\tilde{g}: X_{\tilde{m}}^{u}(\tilde{r}) \rightarrow X_{\tilde{m}}^{c}(\tilde{r})
$$

such that for $\tilde{y}^{u} \in X_{\tilde{m}}^{u}(\tilde{r})$, there exists a unique $y^{u} \in X_{m}^{u}(r)$ such that

$$
D T^{t_{1}}(x)\left(y^{u}+y^{s}+g\left(y^{u}\right)\right)=\tilde{y}^{u}+\tilde{y}^{s}+\tilde{g}\left(\tilde{y}^{u}\right)
$$

where,

$$
\begin{aligned}
& y^{s}=D f_{m}^{c u}\left(x^{u}, x^{c}\right)\left(y^{u}, g\left(y^{u}\right)\right), \\
& \tilde{y}^{s}=D f_{m}^{c u}\left(\tilde{x}^{u}, \tilde{x}^{c}\right)\left(\tilde{y}^{u}, \tilde{g}\left(\tilde{y}^{u}\right)\right) .
\end{aligned}
$$

To do this, for each $\tilde{y}^{u} \in X_{\tilde{m}}^{u}(\tilde{r})$, we define a map $E$ from $\overline{X_{m}^{u}(r)}$ to $X_{m}^{u}$ by

$$
E\left(y^{u}\right)=\left(\left.\Pi_{\tilde{m}}^{u} D T^{t_{1}}(m)\right|_{X_{m}^{u}}\right)^{-1}\left(\tilde{y}^{u}-\Pi_{\tilde{m}}^{u} D T^{t_{1}}(x)\left(y^{u}+y^{s}+g\left(y^{u}\right)\right)\right)+y^{u}
$$

where $y^{s}=D f_{m}^{c u}\left(x^{u}, x^{c}\right)\left(y^{u}, g\left(y^{u}\right)\right)$. In the same fashion as for $E$ in Section 5 , one may show that for small $r, E$ is well defined and a contraction from $\overline{X_{m}^{u}(r)}$ into $X_{m}^{u}(r)$. Thus, $E$ has a unique fixed point $y^{u}$. Let

$$
\tilde{y}^{c}=\Pi_{\tilde{m}}^{c} D T^{t_{1}}(x)\left(y^{u}+y^{s}+g\left(y^{u}\right)\right) .
$$

Define

$$
\tilde{y}^{c}=\tilde{g}\left(\tilde{y}^{u}\right) .
$$

Then, $\tilde{g}$ satisfies the desired conditions. Thus, one can define $\tilde{\gamma}$ by

$$
\tilde{\gamma}(\tilde{x})=[\tilde{g}] \text {. }
$$

The induced graph transform is given by

$$
\mathcal{F}(\gamma)=\tilde{\gamma}
$$

The remaining arguments follow in the same way as those in Section 5. 


\section{REFERENCES}

[An] D. Anosov, Geodesic flows on closed Riemann manifolds with negative curvature, Proc. of the Steklov Inst. of Math., 90, 1967, English translation, Amer. Math. Soc., Providence, R.I. 1969. MR 39:3527

[AG] B. Aulbach and B. M. Garay, Partial linearization for noninvertible mappings, Z. Angew. Math. Phys. 45 (1994), 505-542. MR 95k:58018

[BJ] P. W. Bates and C. K. R. T. Jones, Invariant manifolds for semilinear partial differential equations, Dynamics Reported 2 (1989), 1-38, Wiley. MR 90g:58017

[BL] P. W. Bates and K. Lu, A Hartman-Grobman theorem for the Cahn-Hilliard and phase-field equations, J. Dynamics and Differential Equations 6 (1994), 101-145. MR 94m:35280

[BLZ1] P. W. Bates, K. Lu and C. Zeng, Existence and persistence of invariant manifolds for semiflows in Banach spaces, Mem. Amer. Math. Soc. 135 (1998). MR 99b:58210

[BLZ2] P. W. Bates, K. Lu and C. Zeng, Invariant Manifolds and Invariant Foliations for Semiflows, book, in preparation.

[BDL] A. Burchard, B. Deng and K. Lu, Smooth conjugacy of centre manifolds, Proceedings of the Royal Society of Edingburgh 120A (1992), 61-77. MR 93c:58197

[CHT] X-Y. Chen, J. K. Hale and B. Tan, Invariant foliations for $C^{1}$ semigroups in Banach spaces, J. Differential Equations 139 (1997), 283-318. MR 98m:47109

[CLi] S-N. Chow and X-B. Lin, Bifurcation of a homoclinic orbit with a saddle-node equilibrium, Differential Integral Equations 3 (1990), 435-466. MR 91g:58201

[CLL] S-N. Chow, X-B. Lin and K. Lu, Smooth invariant foliations in infinite dimensional spaces, J. Differential Equations 94 (1991), 266-291. MR 92k:58210

[CL] S-N. Chow and K. Lu, Invariant manifolds and foliations for quasiperiodic systems, J. Differential Equations 117 (1995), 1-27. MR 96b:34064]

[CLM] S-N. Chow, K. Lu and J. Mallet-Paret, Floquet theory for parabolic equations, J. Differential Equations 109 (1994), 147-200. MR 95c:35116

[D1] B. Deng, The Šil'nikov problem, exponential expansion, strong $\lambda$-lemma, $C^{1}$-linearization, and homoclinic bifurcation, J. Differential Equations 82 (1989), 156-173. MR 90k:58161

[D2] B. Deng, The existence of infinitely many traveling front and back waves in the FitzHugh-Nagumo equations, SIAM J. Math. Anal. 22 (1991), 1631-1650. MR 92k:35141

[F1] N. Fenichel, Asymptotic stability with rate conditions, Indiana Univ. Math. Journal 23 (1974), 1109-1137. MR 49:4036

[F2] N. Fenichel, Asymptotic stability with rate conditions. II, Indiana Univ. Math. Journal 26 (1977), 81-93. MR 54:14002

[F3] N. Fenichel, Geometric singular perturbation theory for ordinary differential equations, J. Differential Equations 31 (1979), 53-98. MR 80m:58032

[G] R. A. Gardner, An invariant-manifold analysis of electrophoretic traveling waves, J. Dynamics and Differential Equations 5 (1993), 599-606. MR 94i:34096

[GS] I. Gasser and P. Szmolyan, A geometric singular perturbation analysis of detonation and deflagration waves, SIAM J. Math. Anal. 24 (1993), 968-986. MR 94h:35206

[Ha] J. Hadamard, Sur l'iteration et les solutions asymptotiques des equations differentielles, Bull. Soc. Math. France 29 (1901), 224-228.

[HP] M. W. Hirsch and C. C. Pugh, Stable manifolds and hyperbolic sets, Global Analysis, Proc. Sympos. Pure Math. 14 (1970), 133-163. MR 42:6872

[HPS] M. W. Hirsch, C. C. Pugh and M. Shub, Invariant manifolds, Lecture Notes in Mathematics, vol. 583, Springer-Verlag, New York, 1977. MR 58:18595

[HW] G. Haller and S. Wiggins, N-pulse homoclinic orbits in perturbations of resonant Hamiltonian systems, Arch. Rat. Mech. Anal. 130 (1995), 25-101. MR 96i:34099

[Jo] C. K. R. T. Jones, Geometric singular perturbation theory, C.I.M.E. Lectures (1994).

[JK] C. Jones and N. Kopell, Tracking invariant manifolds with differential forms in singularly perturbed systems, J. Diff. Eq. 108 (1994), 64-88. MR 95c:34085

$[\mathrm{KW}] \quad$ G. Kovacic and S. Wiggins, Orbits homoclinic to resonances with an application to chaos in a model of the forced and damped sine-Gordon equation, Physica-D 57 (1992), 185-225. MR 93f:58153 
[KP] U. Kirchgraber and K. J. Palmer, Geometry in the neighborhood of invariant manifolds of maps and flows and linearization, Pitman Research Notes in Mathematics Series, Longman Scientific and Technical, 1990, John Wiley and Sons, Inc., New York. MR 91k:58115

[Li] X-B. Lin, Shadowing lemma and singularly perturbed boundary value problems, SIAM J. Appl. Math. 49 (1989), 26-54. MR 90a:34126

[LMSW] Y. Li, D. W. McLaughlin, J. Shatah and S. Wiggins, Persistent homoclinic orbits for a perturbed nonlinear Schrödinger equation, Comm. Pure Appl. Math 49 (1996), 11751255. MR 98d:35208

[LW] Y. Li and S. Wiggins, Invariant manifolds and fibrations for perturbed nonlinear Schrödinger equations, Applied Mathematical Sciences, 128, Springer-Verlag, New York, 1997. MR 99e:58165

[Ll] R. de la Llave, Invariant manifolds associated to non-resonant spectral subspaces, J. Statist. Phys. 87 (1997), 211-249. MR 98f:58038

[Lu1] K. Lu, A Hartman-Grobman theorem for scalar reaction-diffusion equations, J. Differential Equations 93 (1991), 364-394. MR 92k:35147]

[Lu2] K. Lu, Structural stability for time periodic parabolic equations, US-Chinese Conference on Differential Equations and their Applications, edited by P. Bates, et al. (1997), 207214. CMP 98:08

[Ly] A. M. Liapunov, Problème géneral de la stabilité du mouvement, Annals Math. Studies 17 (1947).

[Mn] R. Mañé, Lyapunov exponents and stable manifolds for compact transformations, Geometric Dynamics, Lecture Notes in Math., vol. 1007, Springer-Verlag, New York, 1985, pp. 522-577. MR 85j:58126

[Ru] D. Ruelle, Characteristic exponents and invariant manifolds in Hilbert space, Ann. of Math. 115 (1982), 243-290. MR 83j:58097

[Sm] S. Smale, Differentiable dynamical systems, Bull. Amer. Math. Soc. 73 (1967), 747-817.

[Sz] P. Szmolyan, Transversal heteroclinic and homoclinic orbits in singular perturbed problems, J. Differential Equations 92 (1991), 252-281. MR 92m:82141

[Te] D. Terman, The transition from bursting to continuous spiking in excitable membrane models, J. Nonlinear Sci. 2 (1992), 135-182. MR 93g:92008

[W] S. Wiggins, Normally hyperbolic invariant manifolds in dynamical systems, Applied Mathematical Sciences, 105, Springer-Verlag, New York, 1994. MR 95g:58163

Department of Mathematics, Brigham Young University, Provo, Utah 84602

E-mail address: peter@math.byu.edu

Department of Mathematics, Brigham Young University, Provo, Utah 84602

E-mail address: klu@math.byu.edu

Courant Institute of Mathematical Sciences, New York University, New York, New YORK 10012

E-mail address: zengch@math1.cims.nyu.edu 